\title{
Nonadiabatic electron dynamics in time-dependent density-functional theory
}

\author{
C. A. Ullrich ${ }^{1}$ and I. V. Tokatly 2,3 \\ ${ }^{1}$ Department of Physics and Astronomy, University of Missouri, Columbia, Missouri 65211, USA \\ ${ }^{2}$ Lehrstuhl für Theoretische Festkörperphysik, Universität Erlangen-Nürnberg, Staudtstrasse 7/B2, 91058 Erlangen, Germany \\ ${ }^{3}$ Moscow Institute of Electronic Technology, Zelenograd, 124498 Russia
}

(Received 13 February 2006; revised manuscript received 12 April 2006; published 6 June 2006)

\begin{abstract}
Time-dependent density-functional theory (TDDFT) treats dynamical exchange and correlation (xc) via a single-particle potential, $V_{\mathrm{xc}}(\mathbf{r}, t)$, defined as a nonlocal functional of the density $n\left(\mathbf{r}^{\prime}, t^{\prime}\right)$. The popular adiabatic local-density approximation (ALDA) for $V_{\mathrm{xc}}(\mathbf{r}, t)$ uses only densities at the same space-time point $(\mathbf{r}, t)$. To go beyond the ALDA, two local approximations have been proposed based on quantum hydrodynamics and elasticity theory: (a) using the current as the basic variable (C-TDDFT) [G. Vignale, C. A. Ullrich, and S. Conti, Phys. Rev. Lett. 79, 4847 (1997)], (b) working in a comoving Lagrangian reference frame (L-TDDFT) [I. V. Tokatly, Phys. Rev. B 71, 165105 (2005)]. In this paper we illustrate, compare, and analyze both nonadiabatic theories for simple time-dependent model densities in the linear and nonlinear regime, for a broad range of time and frequency scales. C- and L-TDDFT are identical in certain limits, but, in general, exhibit qualitative and quantitative differences in their respective treatment of elastic and dissipative electron dynamics. In situations where the electronic density rapidly undergoes large deformations, it is found that nonadiabatic effects can become significant, causing the ALDA to break down.
\end{abstract}

DOI: 10.1103/PhysRevB.73.235102

PACS number(s): 71.10.-w, 71.15.Mb, 71.45.Gm, 73.21.Fg

\section{INTRODUCTION}

Time-dependent density-functional theory (TDDFT) has gained considerable popularity as a practical approach to the dynamics of many-electron systems. ${ }^{1-4}$ The essential idea of TDDFT is to describe $N$ interacting electrons moving in an external potential $V(\mathbf{r}, t)$ in terms of an auxiliary noninteracting system governed by the time-dependent Kohn-Sham (TDKS) equation,

$$
\left[\frac{1}{i} \frac{\partial}{\partial t}-\frac{\nabla^{2}}{2}+V(\mathbf{r}, t)+V_{\mathrm{H}}(\mathbf{r}, t)+V_{\mathrm{xc}}(\mathbf{r}, t)\right] \varphi_{\alpha}(\mathbf{r}, t)=0
$$

(we use atomic units $\hbar=e=m=1$ throughout). Here and in the following, we consider systems whose ground state and dynamical response are everywhere nonmagnetic, and we may therefore ignore the spin degree of freedom. Equation (1) thus describes the time evolution of doubly occupied Kohn-Sham orbitals $\varphi_{\alpha}(\mathbf{r}, t)$, and the time-dependent density

$$
n(\mathbf{r}, t)=2 \sum_{\alpha=1}^{N / 2}\left|\varphi_{\alpha}(\mathbf{r}, t)\right|^{2}
$$

is obtained in principle exactly. In Eq. (1), $V_{\mathrm{H}}(\mathbf{r}, t)$ $=\int d^{3} r^{\prime} n\left(\mathbf{r}^{\prime}, t\right) /\left|\mathbf{r}-\mathbf{r}^{\prime}\right|$ is the time-dependent Hartree potential, and $V_{\mathrm{xc}}(\mathbf{r}, t)$ is the exchange-correlation (xc) potential. In practice, suitable approximations for $V_{\mathrm{xc}}(\mathbf{r}, t)$ are required. We assume in the following that the system evolves from its ground state at $t=t_{0}$, although this assumption is not strictly necessary.

The exact $V_{\mathrm{xc}}[n](\mathbf{r}, t)$ has a functional dependence on $n(\mathbf{r}, t)$ that is nonlocal in space and time, i.e., contains information about the previous history of the system, including its initial state. ${ }^{5}$ However, almost all present applications of TDDFT employ the adiabatic approximation, ignoring all func- tional dependence of $V_{\mathrm{xc}}$ on prior time-dependent densities $n\left(\mathbf{r}^{\prime}, t^{\prime}\right), t^{\prime}<t$. The simplest example is the adiabatic localdensity approximation (ALDA):

$$
V_{\mathrm{xc}}^{\mathrm{ALA}}(\mathbf{r}, t)=\left.\frac{d \epsilon_{\mathrm{xc}}(\bar{n})}{d \bar{n}}\right|_{\bar{n}=n(\mathbf{r}, t)},
$$

where $\epsilon_{\mathrm{xc}}(\bar{n})$ is the xc energy density of a homogeneous electron gas of density $\bar{n}$. The neglect of retardation in ALDA implies frequency-independent and real xc kernels in the linear response. ${ }^{6}$ This approach has been widely used for calculating molecular excitation energies. ${ }^{7,8}$

One can make the general statement that the adiabatic approximation works well for excitations of the many-body system that have a direct counterpart in the Kohn-Sham system, such as atomic and molecular single-particle excitations. ${ }^{9}$ On the other hand, for more complicated processes such as double or charge-transfer excitations, the ALDA often fails dramatically. ${ }^{10,11}$

Several attempts to go beyond the ALDA can be found in the literature. ${ }^{6,12-19}$ Vignale and Kohn ${ }^{12}$ showed that a nonadiabatic local approximation for exchange and correlation requires the time-dependent current $\mathbf{j}(\mathbf{r}, t)$ as basic variable, rather than the density $n(\mathbf{r}, t)(\mathrm{C}-\mathrm{TDDFT})$. This formalism was later cast in a physically more transparent form using the language of hydrodynamics, ${ }^{13,14}$ where nonadiabatic xc effects appear as viscoelastic stresses in the electron liquid.

To date, C-TDDFT has been applied mainly in frequencydependent linear response. The first application was to calculate linewidths of intersubband plasmons in semiconductor quantum wells. ${ }^{20,21}$ In the absence of disorder and phonon scattering, the ALDA gives infinitely sharp plasmon lines. C-TDDFT includes damping due to electronic many-body effects, in good agreement with experimental linewidths. ${ }^{20,21}$ van Faassen et al..$^{22}$ calculated static axial polarizabilities in molecular chains, which are greatly overestimated with 
ALDA, and achieved an excellent agreement with ab initio quantum chemical results. Other recent studies used C-TDDFT to calculate atomic and molecular excitation energies. ${ }^{23-25}$

Beyond linear response, a wealth of interesting electron dynamics can be explored using TDKS theory. ${ }^{26}$ The C-TDDFT formalism has recently been applied to describe linear and nonlinear charge-density oscillations in quantum wells in the time domain. ${ }^{27}$ It was shown that the retardation caused by the memory dependence of the xc potential has the striking consequence of introducing decoherence and energy relaxation. The mechanism causing this behavior has been discussed by D'Agosta and Vignale. ${ }^{28}$ Formally, dissipation arises in C-TDDFT from a velocity-dependent $\mathrm{xc}$ (vector) potential that breaks the time-reversal invariance of the TDKS Hamiltonian. As a result, a system tends to relax from a nonequilibrium initial state to an equilibrium final state with higher entropy. Because the system is closed and isolated (there is no coupling to a thermal bath), the total energy is conserved. Dissipation in TDKS theory means instead that energy is redistributed between two subsystems with different sets of electronic degrees of freedom, coupled by Coulomb interactions. In the quantum well examples of Refs. 27 and 28, the transfer of energy occurs from a collective motion along the confinement direction into low-lying excitations of the two-dimensional electron gas in the quantum well plane.

A nonadiabatic theory alternative to C-TDDFT has recently been developed by one of the authors. ${ }^{17,18}$ The idea is to relate the local stress in the electron liquid, and thus the xc potential, to the dynamics of deformations of fluid elements in the quantum many-body system. This leads to a formally exact reformulation of TDDFT from the point of view of an observer in a comoving Lagrangian reference frame (LTDDFT). Casting the theory in terms of Lagrangian coordinates allows one to get around the well-known problem of "ultranonlocality" in TDDFT, and to derive, in a rigorous fashion, an exact time-dependent, nonadiabatic extension of the ground-state LDA.

In the L-TDDFT formalism, the xc potential appears as a local functional of the dynamic deformation tensor. At present, two limiting forms of this local functional are available. A high-frequency, "elastic" form of the nonadiabatic xc potential was derived in Ref. 18. The elastic approximation correctly accounts for all complicated nonlinear deformation effects, but completely neglects possible xc contributions to dissipation. The second available limiting form of the nonadiabatic xc potential corresponds to the regime of small deformations. As we will show, in the limit of small deformations L-TDDFT formally reduces to C-TDDFT. In this limit the xc stress (and thus the xc potential) is proportional to the linearized strain tensor that can be considered as a local linear functional of the current. In fact, C-TDDFT of Ref. 13 can be viewed as a viscoelastic linear Hooke's law in the nonlinear quantum continuum mechanics defined by the general formulation of L-TDDFT. In contrast to the purely elastic approximation constructed in Ref. 18, C-TDDFT contains all dissipation effects, but is formally restricted to infinitesimally small deformations. Of course, the formal asymptotic criteria and the practical regimes of applicability of any ap- proximation can be quite different. Therefore a more detailed analysis based on explicit numerical examples is required to assess the validity of the two currently available nonadiabatic xc functionals, and to analyze their relation to the ALDA.

Thus, our purpose in this paper is to illustrate, compare, and analyze C-TDDFT and the elastic approximation to L-TDDFT for simple, quasi-one-dimensional model systems, in order to show the differences and common grounds of both approaches. We explore the performance of the two xc potentials for two kinds of analytically given time-dependent model densities, representing charge-density oscillations in the form of collective sloshing and breathing modes of varying amplitudes and frequencies. This will allow us to simulate electron dynamics over a wide range of time and frequency scales, from the linear to the nonlinear regime. In our analysis, we will focus on a detailed comparison of the timedependent xc potentials, as well as on the instantaneous and time-averaged power absorption associated with the chargedensity oscillations. This will give us insight into the inner workings of C- and L-TDDFT in different dynamical regimes, and their relation to the ALDA. In particular, we will discuss and clarify the meaning of "nonadiabatic," the crossover from the linear to the nonlinear domain, and the competition and coexistence of elastic and dissipative xc effects.

It turns out that, for small-amplitude deformations, C- and L-TDDFT agree in the limit of short time scales or, equivalently, in the high-frequency regime. In general, the two theories show some differences in their treatment of elastic and dissipative effects in the inhomogeneous electron liquid. However, the size of these differences strongly depends on the type of collective mode, and the associated chargedensity deformations. If the deformations are large and occur on short time (high-frequency) scales, both nonadiabatic theories give a clear indication of a failure of the ALDA.

This paper is organized as follows. In Sec. II we summarize the essential formal framework of C-and L-TDDFT. In Sec. III we show how to construct simple analytic model densities in the Lagrangian and associated laboratory reference frame. In Sec. IV we present detailed numerical results and discussion, with a separate treatment of the linear and nonlinear regime. We give our conclusions in Sec. V.

\section{NONADIABATIC THEORIES IN TDDFT}

\section{A. C-TDDFT}

\section{Linear-response regime}

The starting point of C-TDDFT is the linear currentdensity response $\mathbf{j}_{1}(\mathbf{r}, \omega)$ to an external, frequency-dependent vector potential $\mathbf{A}_{\mathrm{ext1}}(\mathbf{r}, \omega)$,

$$
\begin{aligned}
j_{1, i}(\mathbf{r}, \omega)= & \int d^{3} r^{\prime} \chi_{\mathrm{KS}, i j}\left(\mathbf{r}, \mathbf{r}^{\prime}, \omega\right)\left[A_{\mathrm{ext} 1, j}\left(\mathbf{r}^{\prime}, \omega\right)+A_{\mathrm{H} 1, j}\left(\mathbf{r}^{\prime}, \omega\right)\right. \\
& \left.+A_{\mathrm{xc} 1, j}\left(\mathbf{r}^{\prime}, \omega\right)\right],
\end{aligned}
$$

where $i, j$ denote Cartesian coordinates, and $\chi_{\mathrm{KS}, i j}$ is the noninteracting, Kohn-Sham current-current response tensor. Here and in the following, we use the Einstein convention 
for the summation over repeated indices. The Hartree vector potential is given by

$$
A_{\mathrm{H} 1, j}(\mathbf{r}, \omega)=\frac{\nabla_{j}}{(i \omega)^{2}} \int d^{3} r^{\prime} \frac{\nabla^{\prime} \cdot \mathbf{j}_{1}\left(\mathbf{r}^{\prime}, \omega\right)}{\left|\mathbf{r}-\mathbf{r}^{\prime}\right|} .
$$

The simplest approximation for the linearized xc vector potential $\mathbf{A}_{\mathrm{xc} 1}$ is the ALDA, which is defined as

$$
A_{\mathrm{xc} 1, j}^{\mathrm{ALDA}}(\mathbf{r}, \omega)=\frac{\boldsymbol{\nabla}_{j}}{(i \omega)^{2}} \int d^{3} r^{\prime} f_{\mathrm{xc}}^{\mathrm{ALDA}}\left(\mathbf{r}, \mathbf{r}^{\prime}\right) \boldsymbol{\nabla}^{\prime} \cdot \mathbf{j}_{1}\left(\mathbf{r}^{\prime}, \omega\right),
$$

where

$$
f_{\mathrm{xc}}^{\mathrm{ALDA}}\left(\mathbf{r}, \mathbf{r}^{\prime}\right)=\left.\frac{d^{2} \epsilon_{\mathrm{xc}}(\bar{n})}{d \bar{n}^{2}}\right|_{\bar{n}=n_{0}(\mathbf{r})} \delta\left(\mathbf{r}-\mathbf{r}^{\prime}\right)
$$

is the frequency-independent ALDA xc kernel $\left[n_{0}(\mathbf{r})\right.$ is the ground-state density].

Earlier attempts ${ }^{6}$ to go beyond the ALDA and construct a local and frequency-dependent scalar xc potential were unsuccessful since they were found to violate the Harmonic Potential Theorem. ${ }^{29}$ The reason for this failure is that a dynamical xc scalar potential is intrinsically nonlocal in space, i.e., it does not have a gradient expansion in terms of the density. ${ }^{12}$ By contrast, the xc vector potential does admit a frequency-dependent local approximation in terms of the current density. Vignale and $\mathrm{Kohn}^{12}$ derived a currentdensity xc functional in the linear-response regime, assuming that both ground-state and excited-state densities are slowly varying in space. The resulting expression can be written as follows: ${ }^{13,14}$

$$
A_{\mathrm{xc} 1, j}(\mathbf{r}, \omega)=A_{\mathrm{xc} 1, j}^{\mathrm{ALA}}(\mathbf{r}, \omega)-\frac{c}{i \omega n_{0}(\mathbf{r})} \nabla_{k} \sigma_{\mathrm{xc}, j k}(\mathbf{r}, \omega) .
$$

Here, $c$ denotes the speed of light, and $\sigma_{\mathrm{xc}, j k}$ is the xc viscoelastic stress tensor:

$$
\sigma_{\mathrm{xc}, j k}=\eta_{\mathrm{xc}}\left(\boldsymbol{\nabla}_{j} v_{1, k}+\boldsymbol{\nabla}_{k} v_{1, j}-\frac{2}{3} \boldsymbol{\nabla} \cdot \mathbf{v}_{1} \delta_{j k}\right)+\zeta_{\mathrm{xc}} \boldsymbol{\nabla} \cdot \mathbf{v}_{1} \delta_{j k},
$$

where $\mathbf{v}_{1}(\mathbf{r}, \omega)=\mathbf{j}_{1}(\mathbf{r}, \omega) / n_{0}(\mathbf{r})$ is the velocity field associated with the current response, and $\eta_{\mathrm{xc}}$ and $\zeta_{\mathrm{xc}}$ are complex viscosity coefficients defined as

$$
\begin{gathered}
\eta_{\mathrm{xc}}(n, \omega)=-\frac{n^{2}}{i \omega} f_{\mathrm{xc}}^{T}(n, \omega), \\
\zeta_{\mathrm{xc}}(n, \omega)=-\frac{n^{2}}{i \omega}\left(f_{\mathrm{xc}}^{L}(n, \omega)-\frac{4}{3} f_{\mathrm{xc}}^{T}(n, \omega)-\frac{d^{2} \epsilon_{\mathrm{xc}}}{d n^{2}}\right) .
\end{gathered}
$$

$f_{\mathrm{xc}}^{L}(n, \omega)$ and $f_{\mathrm{xc}}^{T}(n, \omega)$ are frequency-dependent xc kernels for the homogeneous electron gas, which can be found in various parametrizations in the literature. ${ }^{6,30,31} \mathrm{In} \mathrm{Eq} .(9), \eta_{\mathrm{xc}}$ and $\zeta_{\mathrm{xc}}$ are both evaluated at the local $n_{0}(\mathbf{r})$.

\section{Nonlinear regime}

The generalization ${ }^{13,27}$ of C-TDDFT into the nonlinear regime and the time domain requires solving the following TDKS equation:

$$
\begin{aligned}
i \frac{\partial \varphi_{\alpha}(\mathbf{r}, t)}{\partial t}= & {\left[\frac{1}{2}\left(\frac{\boldsymbol{\nabla}}{i}+\frac{1}{c} \mathbf{A}(\mathbf{r}, t)+\frac{1}{c} \mathbf{A}_{\mathrm{xc}}(\mathbf{r}, t)\right)^{2}+V(\mathbf{r}, t)\right.} \\
& \left.+V_{\mathrm{H}}(\mathbf{r}, t)\right] \boldsymbol{\varphi}_{\alpha}(\mathbf{r}, t) .
\end{aligned}
$$

Notice that the Hartree term can be expressed as a scalar potential, and we are free to admit external scalar as well as vector potentials.

As explained in Ref. 13, the form of the nonlinear xc vector potential is dictated by a number of general requirements, such as Newton's third law (xc force density follows from a symmetric stress tensor), and the proper limit in the linear regime, which was discussed in the previous subsection. A formally exact, general expression for $\mathbf{A}_{\mathrm{xc}}$ resulting from these requirements will be presented within the Lagrangian framework in Sec. II B. However, a straightforward expression for a nonlinear, nonadiabatic xc vector potential, valid up to second order in the spatial derivatives, follows almost immediately:

$$
\frac{1}{c} \frac{\partial A_{\mathrm{xc}, i}(\mathbf{r}, t)}{\partial t}=-\nabla_{i} V_{\mathrm{xc}}^{\mathrm{ALA}}(\mathbf{r}, t)+\frac{\boldsymbol{\nabla}_{j} \sigma_{\mathrm{xc}, i j}(\mathbf{r}, t)}{n(\mathbf{r}, t)},
$$

where the viscoelastic stress tensor $\sigma_{\mathrm{xc}}$ now contains the time-dependent velocity field $\mathbf{v}(\mathbf{r}, t)=\mathbf{j}(\mathbf{r}, t) / n(\mathbf{r}, t)$ :

$$
\begin{aligned}
\sigma_{\mathrm{xc}, i j}(\mathbf{r}, t)= & \int_{-\infty}^{t} d t^{\prime}\left\{\eta ( \mathbf { r } , t , t ^ { \prime } ) \left[\boldsymbol{\nabla}_{i} v_{j}\left(\mathbf{r}, t^{\prime}\right)+\nabla_{j} v_{i}\left(\mathbf{r}, t^{\prime}\right)\right.\right. \\
& \left.\left.-\frac{2}{3} \boldsymbol{\nabla} \cdot \mathbf{v}\left(\mathbf{r}, t^{\prime}\right) \delta_{i j}\right]+\zeta\left(\mathbf{r}, t, t^{\prime}\right) \boldsymbol{\nabla} \cdot \mathbf{v}\left(\mathbf{r}, t^{\prime}\right) \delta_{i j}\right\} .
\end{aligned}
$$

The viscosity coefficients in Eq. (14) are the Fourier transforms of (10) and (11):

$$
\eta\left(\mathbf{r}, t, t^{\prime}\right)=\left.\int \frac{d \omega}{2 \pi} \eta_{\mathrm{xc}}(\bar{n}, \omega) e^{-i \omega\left(t-t^{\prime}\right)}\right|_{\bar{n}=n(\mathbf{r}, t)},
$$

and similar for $\zeta$. The apparent ambiguity in Eq. (15) whether the density should be evaluated at $t$ or $t^{\prime}$ is resolved by noting that the difference involves higher gradient corrections. We emphasize again that the simple form of Eq. (13) is justified by the basic assumption that the gradients of the density and velocity are small; the velocity itself, on the other hand, need not be small. These points will be elaborated in more detail in Sec. II B, where we will explain how the approximate expression (13) is obtained from the Lagrangian framework in the appropriate limit.

In the following, we consider three-dimensional model systems, uniformly extended along the $y$ and $z$ direction, with a spatial dependence along the $x$ direction only. One can then transform the xc vector potential, Eq. (13), into a scalar one: $V_{\mathrm{xc}}(x, t)=V_{\mathrm{xc}}^{\mathrm{ALDA}}(x, t)+V_{\mathrm{xc}}^{M}(x, t)(\mathrm{ALDA}+M)$, with the memory part given by 


$$
V_{\mathrm{xc}}^{M}(x, t)=-\int_{-\infty}^{x} \frac{d x^{\prime}}{n\left(x^{\prime}, t\right)} \nabla_{x^{\prime}} \sigma_{\mathrm{xc}, x x}\left(x^{\prime}, t\right) .
$$

Assuming that the system has been in the ground state (with zero velocity field) for $t<0$, the $x x$ component of the $\mathrm{xc}$ stress tensor becomes

$$
\sigma_{\mathrm{xc}, x x}\left(x^{\prime}, t\right)=\int_{0}^{t} Y\left(n\left(x^{\prime}, t\right), t-t^{\prime}\right) \boldsymbol{\nabla}_{x^{\prime}} v_{x^{\prime}}\left(x^{\prime}, t^{\prime}\right) d t^{\prime},
$$

where the memory kernel $Y$ is given by

$$
Y\left(n, t-t^{\prime}\right)=\frac{4}{3} \eta\left(n, t-t^{\prime}\right)+\zeta\left(n, t-t^{\prime}\right)
$$

With the help of the Kramers-Kronig relations for $f_{\mathrm{xc}}^{L}$, we can express the memory kernel as follows:

$$
Y\left(n, t-t^{\prime}\right)=\frac{4}{3} \mu_{\mathrm{xc}}-\frac{n^{2}}{\pi} \int \frac{d \omega}{\omega} \Im f_{\mathrm{xc}}^{L}(\omega) \cos \omega\left(t-t^{\prime}\right),
$$

with the xc shear modulus of the electron liquid, ${ }^{31}$

$$
\mu_{\mathrm{xc}}=\frac{3 n^{2}}{4}\left(\mathfrak{R} f_{\mathrm{xc}}^{L}(0)-\frac{d^{2} \epsilon_{\mathrm{xc}}}{d n^{2}}\right) .
$$

The short-time behavior of $Y\left(n, t-t^{\prime}\right)$ is of particular interest, since it governs the high-frequency dynamics. The limit $Y(n, 0)$ can be expressed analytically using the KramersKronig relation,

$$
\int_{-\infty}^{\infty} \frac{d \omega}{\pi} \frac{\mathfrak{I} f_{\mathrm{xc}}^{L}(\omega)}{\omega}=\mathfrak{R} f_{\mathrm{xc}}^{L}(0)-f_{\infty}^{L},
$$

where the high-frequency limit $f_{\infty}^{L}$ is known via the thirdmoment sum rules. ${ }^{31}$ The result is

$$
Y(n, 0)=-\frac{20}{3} \epsilon_{\mathrm{xc}}+\frac{26 n}{5} \frac{d \epsilon_{\mathrm{xc}}}{d n}-\frac{d^{2} \epsilon_{\mathrm{xc}}}{d n^{2}} .
$$

It is also straightforward to see that $(d Y / d t)(n, 0)=0$, i.e., the memory kernel starts with zero slope.

Evaluation of the full memory kernel $Y\left(n, t-t^{\prime}\right)$ requires $f_{\mathrm{xc}}^{L}(\omega)$ as input, which to date is only available in terms of approximate interpolation formulas based on known exact properties and sum rules. ${ }^{9}$ The older Gross-Kohn $(\mathrm{GK})^{6}$ parametrization of $f_{\mathrm{xc}}^{L}(\omega)$ by construction satisfies the exact high-frequency limit; the more recent Qian-Vignale $(\mathrm{QV})^{31}$ parametrization, in addition, has the correct low-frequency behavior and accounts for the presence of the two-plasmon peak at intermediate frequencies. ${ }^{30}$

Figure 1 shows the memory kernel $Y\left(n, t-t^{\prime}\right)$ evaluated with the GK and QV parametrizations for $f_{\mathrm{xc}}^{L}(\omega)$, and scaled by the short-time limit $Y(n, 0)$ (see the inset). Here, $T_{p}$ $=2 \pi / \omega_{p}$ is the characteristic time scale associated with the plasma frequency $\omega_{p}=\sqrt{4 \pi n}$. As noted earlier, ${ }^{27} Y^{\mathrm{GK}}$ and $Y^{\mathrm{QV}}$ are identical at $t=t^{\prime}$ and have a similar short-time behavior, since both approaches satisfy the correct highfrequency limit for $f_{\mathrm{xc}}^{L}(\omega)$. $Y^{\mathrm{GK}}$ goes exponentially to zero for large $t-t^{\prime}$, and the scaled GK memory kernels are nearly

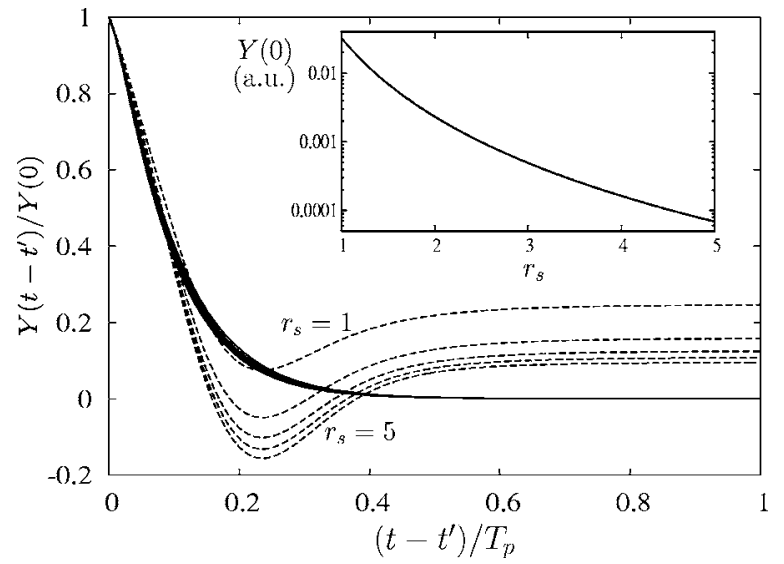

FIG. 1. Scaled memory kernel $Y\left(n, t-t^{\prime}\right)$ for $r_{s}=1,2,3,4,5$, using the GK (full lines) and QV (dashed lines) parametrizations. Inset: short-time limit $Y(n, 0)$ [Eq. (22)] versus $r_{s}$.

identical for all times over a wide range of $r_{s}$. On the other hand, all $Y^{\mathrm{QV}}$ pass through a minimum around $0.22 T_{p}$ and then approach the finite limit $4 \mu_{\mathrm{xc}} / 3$, where $\mu_{\mathrm{xc}} \rightarrow 0$ for large $r_{s}{ }^{31}$

It is interesting to notice that both GK and QV memory kernels reach their long-time asymptotic limits very quickly, within about half a plasma cycle $T_{p}$. This rapid initial decrease of $Y\left(n, t-t^{\prime}\right)$ can be interpreted as loss of memory of the electron gas, which has the consequence of introducing dissipation in the electron dynamics, as we will see later. The long-time behaviors, on the other hand, are connected to the zero-frequency limits of $f_{\mathrm{xc}}^{L}(\omega)$, which are different in the GK and QV parametrizations. The consequences of this difference will be explored in Sec. IV A.

\section{B. L-TDDFT}

\section{General formulation}

The main problem in constructing nonadiabatic approximations for xc potentials is an inherent nonlocality of the time-dependent theory. Physically, this nonlocality is related to the convective motion of the electron fluid (the particles at a given point of space retain the memory of their previous positions). ${ }^{18}$ The key idea of L-TDDFT is to eliminate the above source of nonlocality by reformulating the theory in the Lagrangian frame, i.e., in a local reference frame moving with the fluid. Since the convective motion in the Lagrangian frame is absent, a spatially local description of xc effects becomes possible. This possibility represents the most important outcome of L-TDDFT: it allows one to derive an exact nonadiabatic extension of the LDA into the dynamic regime.

The general formulation of L-TDDFT starts with the exact relation of the xc force to the xc stress tensor $P_{\mathrm{xc}, i j} \cdot{ }^{16,18}$ By definition, the xc vector potential $\mathbf{A}_{\mathrm{xc}}$ ensures that the physical density and current are reproduced by an auxiliary system of noninteracting $\mathrm{KS}$ particles. This means that $\mathbf{A}_{\mathrm{xc}}$ should produce an effective xc Lorentz force that exactly compensates for a difference of local stress forces in the real interacting system and in the noninteracting KS system. Ac- 
cordingly, the xc vector potential should satisfy the following equation:

$$
-\frac{\partial A_{\mathrm{xc}, i}}{\partial t}+v_{j}\left(\nabla_{i} A_{\mathrm{xc}, j}-\nabla_{j} A_{\mathrm{xc}, i}\right)=\frac{c}{n} \nabla_{j} P_{\mathrm{xc}, i j},
$$

where $P_{\mathrm{xc}, i j}=P_{i j}-T_{i j}^{\mathrm{KS}}$ is the difference of the full stress tensor for the interacting system, $P_{i j}$, and the kinetic stress tensor for the KS system, $T_{i j}^{\mathrm{KS}}$. Equation (23) serves as a basic definition of $\mathbf{A}_{\mathrm{xc}}$, which automatically accounts for the zero force and zero torque conditions. ${ }^{12}$

We note that the xc stress tensor $P_{\mathrm{xc}, i j}$ is to be distinguished from the earlier introduced xc stress tensor $\sigma_{\mathrm{xc}, i j}$. The connection between the two will be explained in detail in Sec. II B 2. The main difference lies in the fact that $P_{\mathrm{xc}, i j}$, formally exactly and to all orders in the inhomogeneity, accounts for all dynamical xc effects, whereas the ALDA has been separated out in the definition of $\sigma_{\mathrm{xc}, i j}$. Furthermore, $\sigma_{\mathrm{xc}, i j}$ is valid only for small deformations of the electron liquid (in a sense to be defined later).

All the rest of L-TDDFT can be viewed as a calculation of the dynamic xc stress tensor $P_{\mathrm{xc}, i j}$, which enters the definition of $\mathbf{A}_{\mathrm{xc}}$, Eq. (23), by reformulating the problem in the comoving Lagrangian frame. The transformation to the Lagrangian frame corresponds to a nonlinear transformation of coordinates, $\mathbf{r}=\mathbf{r}(\boldsymbol{\xi}, t)$, where $\mathbf{r}(\boldsymbol{\xi}, t)$ is the trajectory of an infinitesimal fluid element that evolves from the point $\boldsymbol{\xi}$. Formally the function $\mathbf{r}(\boldsymbol{\xi}, t)$ is defined by the following initial value problem:

$$
\frac{\partial \mathbf{r}(\boldsymbol{\xi}, t)}{\partial t}=\mathbf{v}(\mathbf{r}(\boldsymbol{\xi}, t), t), \quad \mathbf{r}(\boldsymbol{\xi}, 0)=\boldsymbol{\xi} .
$$

The initial positions, $\boldsymbol{\xi}$, of the fluid elements play the role of spatial coordinates in the comoving frame. The previous transformation from the old coordinates $\mathbf{r}$ to the new coordinates $\boldsymbol{\xi}$ induces a change of metric. The metric tensor in the Lagrangian $\boldsymbol{\xi}$ space coincides with the Green's deformation tensor,

$$
g_{i j}(\boldsymbol{\xi}, t)=\frac{\partial r_{k}(\boldsymbol{\xi}, t)}{\partial \xi_{i}} \frac{\partial r_{k}(\boldsymbol{\xi}, t)}{\partial \xi_{j}}
$$

which is a common characteristic of deformations in the Lagrangian formulation of continuum mechanics. A complete reformulation of TDDFT in the comoving frame shows that a local description of xc effects is possible if one uses $g_{i j}(\xi, t)$ as a basic variable: the stress tensor in the Lagrangian frame can be consistently considered as a spatially local functional of the Green's deformation tensor. ${ }^{32}$

For most practical applications we need the xc potential in the laboratory frame, Eq. (23). Transforming the stress tensor functional from the comoving to the laboratory frame, we find the required tensor $P_{\mathrm{xc}, i j}$ entering Eq. (23). The locality in the Lagrangian frame translates to a local dependence of $P_{\mathrm{xc}, i j}$ on the Cauchy's deformation tensor,

$$
\bar{g}_{i j}(\mathbf{r}, t)=\frac{\partial \xi_{k}(\mathbf{r}, t)}{\partial r_{i}} \frac{\partial \xi_{k}(\mathbf{r}, t)}{\partial r_{j}},
$$

where the function $\xi(\mathbf{r}, \mathbf{t})$ is obtained by inverting the trajectory equation, $\mathbf{r}=\mathbf{r}(\boldsymbol{\xi}, t)$. The Cauchy's tensor $\bar{g}_{i j}(\mathbf{r}, t)$ is commonly used to describe deformations in the Eulerian formulation of continuum mechanics. From a physical point of view, the functional dependence of the stress tensor on $\bar{g}_{i j}$ thus emerges quite naturally.

The substitution of $P_{\mathrm{xc}, i j}\left[\bar{g}_{i j}\right]$ into Eq. (23) yields the xc vector potential $\mathbf{A}_{\mathrm{xc}}$ as a functional of Cauchy's deformation tensor. If gradients of the deformation tensor are small, the functional $P_{\mathrm{xc}, i j}\left[\bar{g}_{i j}\right]$ is local in space, but, in general, it can be nonlocal in time. The time nonlocality of the stress tensor functional breaks time-reversal invariance, similar to the velocity-dependent xc potential in C-TDDFT (see the discussion in the Introduction), leading to dissipation and thus a loss of memory. In the absence of dissipation (e.g., in the exchange-only approximation), the stress tensor becomes a simple function of $\bar{g}_{i j}$, which corresponds to an infinitely long memory.

Equations (24) and (26) determine Cauchy's deformation tensor as a functional of velocity $\mathbf{v}(\mathbf{r}, t)$. An alternative, and practically more convenient way to compute $\bar{g}_{i j}$ for a given velocity is to solve the equation of motion that governs the dynamics of $\bar{g}_{i j}(\mathbf{r}, t)$ directly in the laboratory frame:

$$
\frac{\partial \bar{g}_{i j}}{\partial t}+v_{k} \frac{\partial \bar{g}_{i j}}{\partial r_{k}}=-\frac{\partial v_{k}}{\partial r_{i}} \bar{g}_{k j}-\frac{\partial v_{k}}{\partial r_{j}} \bar{g}_{i k}, \quad \bar{g}_{i j}(\mathbf{r}, 0)=\delta_{i j} .
$$

An important property of the deformation tensor is that it allows us to relate the time-dependent density $n(\mathbf{r}, t)$ to the initial density distribution, $n_{0}(\mathbf{r})$ :

$$
n(\mathbf{r}, t)=\sqrt{\bar{g}(\mathbf{r}, t)} n_{0}(\boldsymbol{\xi}(\mathbf{r}, \mathbf{t})),
$$

where $\bar{g}(\mathbf{r}, t)$ is the determinant of $\bar{g}_{i j}(\mathbf{r}, t)$.

Equation (27) or, equivalently, Eqs. (24) and (26), show that, in general, the deformation tensor is a strongly nonlocal (both in space and in time) functional of the velocity. Therefore, in spite of the fact that the xc stress tensor and consequently the xc vector potential are local functionals of $\bar{g}_{i j}$, they are nonlocal in terms of velocity or any other variable. This makes the choice of $\bar{g}_{i j}(\mathbf{r}, t)$ as a basic variable much more preferable.

To obtain an explicit construction of the local functional $P_{\mathrm{xc}, i j}\left[\bar{g}_{i j}\right]$, a solution of a homogeneous time-dependent many-body problem in the Lagrangian frame is required (see Sec. V in Ref. 18). A complete solution of this problem seems to be impossible, at least at the current level of knowledge. However, there are two practically important, exactly solvable special cases, which are described in the following.

\section{Small deformation approximation: Recovery of $C$ - TDDFT}

The above-mentioned many-body problem in the homogeneously deformed Lagrangian $\boldsymbol{\xi}$ space can be solved perturbatively if the deformation tensor $\bar{g}_{i j}$ only slightly deviates from the unit tensor $\delta_{i j}$ : 


$$
\bar{g}_{i j}(\mathbf{r}, t)=\delta_{i j}+\delta \bar{g}_{i j}(\mathbf{r}, t) .
$$

Introducing the displacement vector, $\mathbf{u}(\mathbf{r}, t)=\mathbf{r}-\boldsymbol{\xi}(\mathbf{r}, t)$, and using Eq. (26), we find that small $\delta \bar{g}_{i j}$ corresponds to small gradients of the displacement:

$$
\delta \bar{g}_{i j}(\mathbf{r}, t)=-\left(\frac{\partial u_{i}}{\partial r_{j}}+\frac{\partial u_{j}}{\partial r_{i}}\right)
$$

Clearly, small gradients of $\mathbf{u}(\mathbf{r}, t)$ imply that the velocity gradients are also small, since to lowest order in $\nabla_{i} u_{j}$ Eq. (24) reduces to the relation $\partial_{t} \mathbf{u}(\mathbf{r}, t)=\mathbf{v}(\mathbf{r}, t)$. Obviously the smallness of deformations does not mean that the displacement or the velocity themselves are small (i.e., the system can be far beyond the linear response regime). A well known example is the rigid motion of a many-body system in a harmonic potential, where $\bar{g}_{i j}=\delta_{i j}$, but the displacement can be arbitrarily large.

The stress tensor functional for small displacement vectors was derived in Ref. 18. An extension of this derivation to the general regime of small deformations, i.e., to the regime of small displacement gradients, is straightforward. The resulting $\mathrm{xc}$ stress tensor takes the following form:

$$
P_{\mathrm{xc}, i j}(\mathbf{r}, t)=P_{\mathrm{xc}}^{\mathrm{ALDA}}(n(\mathbf{r}, t)) \delta_{i j}+\delta P_{i j}(\mathbf{r}, t),
$$

where $P_{\mathrm{xc}}^{\mathrm{ALA}}(n)$ is the xc pressure of a homogeneous electron gas, and $\delta P_{i j}$ is a nonadiabatic correction, which is linear in $\delta \bar{g}_{i j}$ :

$$
\begin{aligned}
\delta P_{i j}(\mathbf{r}, t)= & \int_{0}^{t} d t^{\prime}\left[\frac{\delta_{i j}}{2} \tilde{K}_{\mathrm{xc}}\left(n(\mathbf{r}, t), t-t^{\prime}\right) \delta \bar{g}_{k k}\left(\mathbf{r}, t^{\prime}\right)\right. \\
& \left.+\mu_{\mathrm{xc}}\left(n(\mathbf{r}, t), t-t^{\prime}\right)\left(\delta \bar{g}_{i j}\left(\mathbf{r}, t^{\prime}\right)-\frac{\delta_{i j}}{3} \delta \bar{g}_{k k}\left(\mathbf{r}, t^{\prime}\right)\right)\right] .
\end{aligned}
$$

The kernels $\mu_{\mathrm{xc}}\left(n, t-t^{\prime}\right)$ and $\widetilde{K}_{\mathrm{xc}}\left(n, t-t^{\prime}\right)$ in Eq. (32) have the meaning of nonadiabatic shear and bulk moduli, respectively [the adiabatic part of the bulk modulus is included in the ALDA pressure term in Eq. (31)]. The corresponding Fourier transforms of the elastic moduli, $\mu_{\mathrm{xc}}(n, \omega)$ and $\widetilde{K}_{\mathrm{xc}}(n, \omega)$, are related to the complex viscosity coefficients of Eqs. (10) and (11), $\eta_{\mathrm{xc}}(n, \omega)$ and $\zeta_{\mathrm{xc}}(n, \omega)$, as follows:

$$
\mu_{\mathrm{xc}}(\omega)=-i \omega \eta_{\mathrm{xc}}(\omega), \quad \tilde{K}_{\mathrm{xc}}(\omega)=-i \omega \zeta_{\mathrm{xc}}(\omega) .
$$

Using Eq. (33) and the relation $\partial_{t} \mathbf{u}=\mathbf{v}$, we find that the nonadiabatic stress tensor $\delta P_{i j}$, Eq. (32), is identical, up to a sign, ${ }^{33}$ to the tensor $\sigma_{\mathrm{xc}, i j}$ of Eq. (14) [i.e., $\left.\delta P_{i j}=-\sigma_{\mathrm{xc}, i j}\right]$. In addition, in the limit of small displacement/velocity gradients, the spatial derivatives of $\mathbf{A}_{\mathrm{xc}}$ on the left hand side of Eq. (23) are negligible. Thus, in the regime of small deformations we recover the complete nonlinear form of C-TDDFT, ${ }^{13,27}$ Eqs. (13) and (14).

The imaginary parts of the complex elastic moduli, $\tilde{K}_{\mathrm{xc}}(\omega)$ and $\mu_{\mathrm{xc}}(\omega)$, are responsible for the dissipative (viscous) effects. For the high-frequency/short-time dynamics these effects become irrelevant. As a result, the highfrequency limit of the nonadiabatic stress tensor of Eq. (32) becomes completely local and purely elastic:

$$
\begin{aligned}
\delta P_{i j}^{\infty}(\mathbf{r}, t)= & \frac{\delta_{i j}}{2} \tilde{K}_{\mathrm{xc}}^{\infty}(n(\mathbf{r}, t)) \delta \bar{g}_{k k}(\mathbf{r}, t)+\mu_{\mathrm{xc}}^{\infty}(n(\mathbf{r}, t)) \\
& \times\left(\delta \bar{g}_{i j}(\mathbf{r}, t)-\frac{\delta_{i j}}{3} \delta \bar{g}_{k k}(\mathbf{r}, t)\right),
\end{aligned}
$$

where $\tilde{K}_{\mathrm{xc}}^{\infty}(n)$ and $\mu_{\mathrm{xc}}^{\infty}(n)$ are the high-frequency limits of the bulk and shear moduli, respectively.

The structure of the small deformation approximation, Eqs. (31) and (32), clearly demonstrates that in this regime the nonadiabatic contribution appears as a small, linear in $\delta \bar{g}_{i j}$ correction to the adiabatic dynamics. If the process is strongly nonadiabatic, the deformations cannot be considered small. In fact, the deviation of the deformation tensor from $\delta_{i j}$ can serve as a general measure of nonadiabaticity.

\section{Nonlinear elastic approximation to L-TDDFT}

It is very difficult to account both for the full nonlinear dependence on $\bar{g}_{i j}$ and for the dissipation. C-TDDFT includes all xc dissipation effects on a level linear in $\delta \bar{g}_{i j}$. On the contrary, if we neglect the dissipation effects, a closed nonlinear local approximation for the stress tensor can be rigorously derived. ${ }^{18}$ The reason is that the homogeneous many-body problem, which has been formulated in Ref. 18, admits a simple complete solution in the regime of fast dynamics when the dissipation is irrelevant. In this case the $\mathrm{xc}$ stress tensor $P_{\mathrm{xc}, i j}(\mathbf{r}, t)$ becomes a function of the timedependent density $n(\mathbf{r}, t)$ and the Cauchy's deformation tensor $\bar{g}_{i j}(\mathbf{r}, t)$ :

$$
P_{\mathrm{xc}, i j}=\frac{2}{3} \bar{g}_{i j} \sqrt{\bar{g}} E_{\mathrm{kin}}^{\mathrm{xc}}\left(\frac{n}{\sqrt{\bar{g}}}\right)+L_{i j}\left(\bar{g}_{k l}\right) E_{\mathrm{pot}}\left(\frac{n}{\sqrt{\bar{g}}}\right),
$$

where $E_{\mathrm{kin}}^{\mathrm{xc}}(n)$ and $E_{\mathrm{pot}}(n)$ are the xc kinetic and potential energy per unit volume of a homogeneous electron gas, which, for a three-dimensional system, are given by ${ }^{34}$

$$
\begin{gathered}
E_{\mathrm{kin}}^{\mathrm{xc}}(n)=3 n^{7 / 3}\left(\frac{\epsilon_{\mathrm{xc}}}{n^{4 / 3}}\right)^{\prime}, \\
E_{\mathrm{pot}}^{\mathrm{xc}}(n)=-3 n^{8 / 3}\left(\frac{\epsilon_{\mathrm{xc}}}{n^{5 / 3}}\right)^{\prime} .
\end{gathered}
$$

The function $L_{i j}\left(\bar{g}_{k l}\right)$ in Eq. (35) is explicitly defined in Appendix A of Ref. 18. In the limit of small deformations, the nonlinear elastic approximation of Eq. (35) can be expanded around $\bar{g}_{i j}=\delta_{i j}$ and reduces to the linearized form defined by Eqs. (31) and (34). In other words, one recovers the highfrequency limit of C-TDDFT.

We conclude this section with the explicit formulation of the nonlinear elastic approximation for a one-dimensional motion. If all spatial variations are along the $x$ axis only, the deformation tensor takes a diagonal form with $\bar{g}_{z z}=\bar{g}_{y y}=1$, and $\bar{g}_{x x}=\bar{g}(x, t)$. The xc effects can then be described by an $\mathrm{xc}$ scalar potential that is related to the $\mathrm{xc}$ stress tensor as follows: 


$$
V_{\mathrm{xc}}^{E}(x, t)=\int_{-\infty}^{x} \frac{d x^{\prime}}{n\left(x^{\prime}, t\right)} \frac{\partial}{\partial x^{\prime}} P_{\mathrm{xc}, x x}\left(n\left(x^{\prime}, t\right), \bar{g}\left(x^{\prime}, t\right)\right) .
$$

Equation (35) for the $x x$ component of the $x c$ stress tensor reduces to the form

$$
P_{\mathrm{xc}, x x}(n, \bar{g})=\frac{2}{3} \bar{g}^{3 / 2} E_{\mathrm{kin}}^{\mathrm{xc}}\left(\frac{n}{\sqrt{\bar{g}}}\right)+L(\bar{g}) E_{\mathrm{pot}}\left(\frac{n}{\sqrt{\bar{g}}}\right),
$$

where the factor $L(\bar{g})$ is given by

$$
L(\bar{g})=\frac{\bar{g}}{\bar{g}-1}\left(1-\frac{\arctan \sqrt{\bar{g}-1}}{\sqrt{\bar{g}-1}}\right) .
$$

Finally, Eq. (27), which relates the deformation $\bar{g}(x, t)$ to the velocity $v(x, t)$, simplifies as follows:

$$
\frac{\partial \bar{g}}{\partial t}=-v \frac{\partial \bar{g}}{\partial x}-2 \frac{\partial v}{\partial x} \bar{g}, \quad \bar{g}(x, 0)=1 .
$$

It is worth mentioning that $L(\bar{g} \rightarrow 1)=1 / 3$ in the limit of zero deformation. Equation (39) then approaches the standard virial expression for the xc pressure, and $V_{\mathrm{xc}}^{E}(x, t)$ reduces to the ALDA xc potential. We define the post-ALDA contribution of the nonlinear elastic L-TDDFT xc potential as

$$
\tilde{V}_{\mathrm{xc}}^{E}(x, t)=V_{\mathrm{xc}}^{E}(x, t)-V_{\mathrm{xc}}^{\mathrm{ALDA}}(x, t) .
$$

To shorten the notation for the rest of this paper: from now on, whenever we refer to L-TDDFT, we actually mean the nonlinear elastic approximation to L-TDDFT, defined in this subsection.

\section{CONSTRUCTION OF ONE-DIMENSIONAL ANALYTICAL EXAMPLES}

In one dimension, the trajectory of a fluid element with Lagrangian coordinate $\xi$ is determined by the following equation:

$$
\frac{\partial x(\xi, t)}{\partial t}=v(x(\xi, t), t), \quad x(\xi, 0)=\xi
$$

$x$ is the position, at time $t$, of a fluid element that started out at $\xi$, and $v$ is its velocity. In general, this is a complicated nonlinear differential equation, with formal solution

$$
x(\xi, t)=\xi+\int_{0}^{t} v\left(\xi, t^{\prime}\right) d t^{\prime} .
$$

In other words, if we know the velocity, at time $t$, of a fluid element that started out at $\xi$, we can determine its trajectory by direct integration. From this, we can then determine the time-dependent density in the laboratory frame: we first invert (44) to obtain $\xi(x, t)$; then compute the deformation as

$$
\bar{g}(x, t)=\left(\frac{\partial \xi}{\partial x}\right)^{2}
$$

and finally obtain

$$
n(x, t)=\sqrt{\bar{g}(x, t)} n_{0}(\xi(x, t)) .
$$

In practice, of course, this procedure is not very helpful, since the functional form of $v(\xi, t)$ is unknown. However, we can use it to construct simple analytic examples, as follows.

\section{A. Sloshing mode}

We assume that the system is confined within hard walls, $-L / 2 \leqslant(x, \xi) \leqslant L / 2$, with initial density

$$
n_{0}(\xi)=\frac{2 N}{L} \cos ^{2}\left(\frac{\pi \xi}{L}\right)
$$

where $N$ is the number of electrons per unit area (sheet density) in the $y-z$ plane. We assume a simple quadratic form of the velocity field:

$$
v(\xi, t)=A \omega\left(\frac{L}{4}-\frac{\xi^{2}}{L}\right) \cos \omega t .
$$

Equation (44) is then easily integrated:

$$
x(\xi, t)=\xi+A\left(\frac{L}{4}-\frac{\xi^{2}}{L}\right) \sin \omega t .
$$

The next step is to invert Eq. (49) to determine the trajectories of the fluid elements, which requires solving a quadratic equation, with the result

$$
\xi(x, t)=\frac{L}{2 A \sin \omega t}\left(1-\sqrt{1+A^{2} \sin ^{2} \omega t-\frac{4 A x}{L} \sin \omega t}\right),
$$

which properly reduces to $\xi=x$ for $A \rightarrow 0$. The range of allowed amplitudes is $|A| \leqslant 1$, which is dictated by the constraint that no fluid element can cross the hard-wall boundaries at $\pm L / 2$.

We can now calculate the deformation using Eq. (45):

$$
\bar{g}(x, t)=\left(1+A^{2} \sin ^{2} \omega t-\frac{4 A x}{L} \sin \omega t\right)^{-1} .
$$

The time-dependent density of the sloshing mode in the laboratory frame, $n(x, t)$, then follows from Eq. (46), using (50) and (51).

\section{B. Breathing mode}

To simulate a breathing mode, we assume a linear velocity distribution of the fluid elements:

$$
v(\xi, t)=A \omega \xi \cos \omega t
$$

According to Eq. (44), this gives the following trajectory:

$$
x(\xi, t)=\xi(1+A \sin \omega t), \quad|A|<1 .
$$

This is easily inverted:

$$
\xi(x, t)=\frac{x}{1+A \sin \omega t},
$$

and the resulting deformation is 


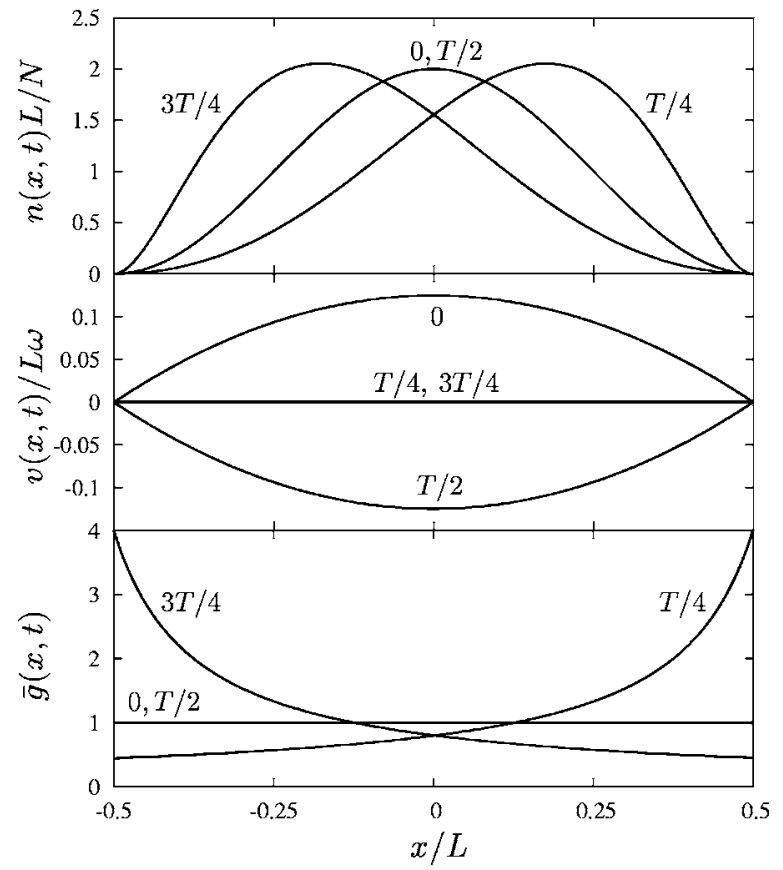

FIG. 2. Snapshots of density $n(x, t)$, in units of $N / L$, velocity $v(x, t)$, in units of $L \omega$, and deformation $\bar{g}(x, t)$ for the sloshing mode in the laboratory frame, taken at times $t=0, T / 4, T / 2,3 T / 4$.

$$
\bar{g}(x, t)=\frac{1}{(1+A \sin \omega t)^{2}} .
$$

We choose the same initial density distribution $n_{0}(\xi)$, Eq. (47), as for the sloshing mode, and the resulting timedependent density of the breathing mode is

$$
n(x, t)=\frac{2 N}{L} \cos ^{2}\left(\frac{\pi x / L}{1+A \sin \omega t}\right)(1+A \sin \omega t)^{-1},
$$

where $|x|<(L / 2)(1+A \sin \omega t)$.

Figures 2 and 3 show snapshots of $n(x, t), v(x, t)$, and $\bar{g}(x, t)$ for the sloshing and the breathing mode, taken at time $t=0, T / 4, T / 2,3 T / 4$, where $T=2 \pi / \omega$. The amplitude is $A$ $=0.5$ in both cases, length is measured in units of $L$, and density and velocity are plotted in units of $N / L$ and $L \omega$, respectively. The deformation $\bar{g}(x, t)$ is maximal at the turning points of the oscillations (at $t=T / 4$ and 3T/4). We find that the breathing mode features large deformations everywhere, i.e., $\bar{g}$ deviates strongly from one. The sloshing mode, on the other hand, is strongly deformed only toward the edges, where the density is small. We will see below how this affects the nonadiabaticity of the xc potential of the two modes.

\section{RESULTS AND DISCUSSION}

In the following numerical examples, we choose a system where, in atomic units, we have sheet density $N=1$ a.u. and quantum well width $L=10$ a.u. We shall measure frequencies in units of the average plasmon frequency of the system, given by

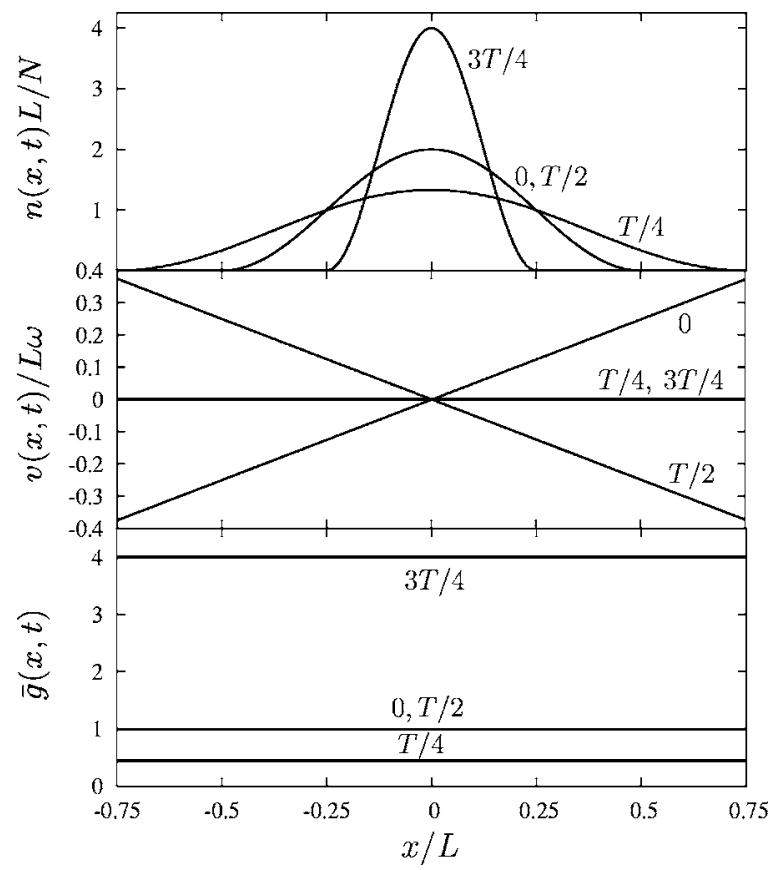

FIG. 3. The same as Fig. 2, for the breathing mode.

$$
\bar{\omega}_{p}=\frac{1}{L} \int d x \omega_{p}(n(x)) .
$$

For our initial density distribution $n_{0}(x)$, Eq. (47), we find $\bar{\omega}_{p}=\sqrt{32 N / \pi L}$, which for the above values of $N$ and $L$ comes out as $\bar{\omega}_{p}=1.009$ a.u.

\section{A. Linear regime}

\section{Time-dependent xc potentials}

We first consider the linear regime of small density fluctuations. For the time-dependent densities associated with the breathing mode and the sloshing mode, we calculate and compare three different time-dependent xc potentials: $V_{\mathrm{xc}}^{M}(x, t)$ from C-TDDFT [Eq. (16)], $\tilde{V}_{\mathrm{xc}}^{E}(x, t)$ from L-TDDFT [Eq. (42)], and the ALDA potential fluctuations $\widetilde{V}_{\mathrm{xc}}^{\mathrm{ALA}}(x, t)$ $=V_{\mathrm{xc}}^{\mathrm{ALDA}}(n(x, t))-V_{\mathrm{xc}}^{\mathrm{LDA}}\left(n_{0}(x)\right)$.

Figures 4-7 each show four snapshots of $V_{\mathrm{xc}}^{M}(x, t)$ and $\tilde{V}_{\mathrm{xc}}^{\mathrm{ALDA}}$ during one cycle of the sloshing/breathing modes, at $t=0, T$ and $T / 2$ (density passes through equilibrium, with maximal velocity) and $t=T / 4$ and $3 T / 4$ (density at turning points), as illustrated in Figs. 2 and $3 . V_{\mathrm{xc}}^{M}(x, t)$ has been calculated using the $\mathrm{GK}$ and $\mathrm{QV}$ parametrizations as input (Figs. 4 and 6 and Figs. 5 and 7 respectively). All density oscillations have the same small amplitude $A=0.005$, but different frequencies. We highlight the low- and high-frequency limit, $\omega_{L}=0.001 \bar{\omega}_{p}$ and $\omega_{H}=1000 \bar{\omega}_{p}$, respectively, and an intermediate crossover frequency $\omega_{C}$, to be defined later, which varies between $1.7 \bar{\omega}_{p}$ and $2.65 \bar{\omega}_{p}$ for the four cases considered. To illustrate the strong dependence on frequency in the crossover regime, we also show $V_{\mathrm{xc}}^{M}$ at $0.1,0.5,2$, and 10 times $\omega_{C}$. In the high-frequency limit $\omega_{H}, V_{\mathrm{xc}}^{M}(x, t)$ is identical to $\widetilde{V}_{\mathrm{xc}}^{E}(x, t)$ in all cases, i.e., the dynamics is purely elastic. 


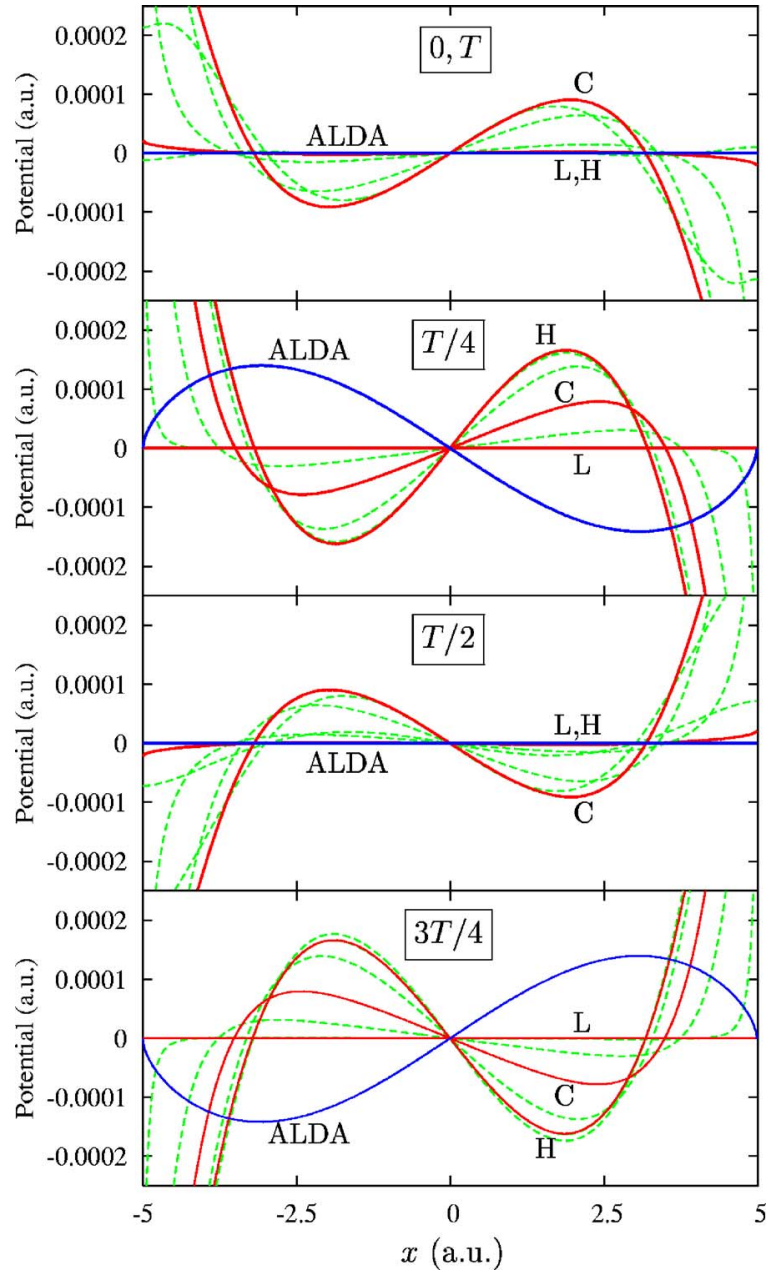

FIG. 4. (Color online) Snapshots of ALDA xc potential fluctuations and xc memory contributions during one cycle of the sloshing mode (Fig. 2), with amplitude $A=0.005$. Blue (dark gray) solid lines: $\widetilde{V}_{\text {xc }}^{\text {ALDA }}$, scaled by 0.1 (independent of frequency). Red (medium gray) solid lines: $V_{\mathrm{xc}}^{M}$, in GK parametrization, for low $(L)$ frequency, $\omega_{L}=0.001 \bar{\omega}_{p}$, crossover $(C)$ frequency, $\omega_{C}=1.7 \bar{\omega}_{p}$, and high $(H)$ frequency, $\omega_{H}=1000 \bar{\omega}_{p}$. Green (light gray) dashed lines: $V_{\mathrm{xc}}^{M}$ at $0.1,0.5,2,10$ times $\omega_{C}$.

The results in Figs. 4-7 reveal the following features.

(i) The high-frequency limit of $V_{\mathrm{xc}}^{M}(x, t)$ [i.e., $\left.\widetilde{V}_{\mathrm{xc}}^{E}(x, t)\right]$ is phase shifted by $\pi$ with respect to $\widetilde{V}_{\mathrm{xc}}^{\mathrm{ALDA}}(x, t)$ in all cases considered. This is to be expected for a purely elastic potential: it reaches its maximum at the instant of largest displacement from equilibrium.

(ii) At the low-frequency limit $\omega_{L}$, the behavior of $V_{\mathrm{xc}}^{M}$ depends on the parametrization used to calculate the memory kernel: in $\mathrm{GK}, V_{\mathrm{xc}}^{M}$ vanishes, whereas in $\mathrm{QV}, V_{\mathrm{xc}}^{M}$ again becomes purely elastic. This reflects the different long-time behaviors of the memory kernel: in GK, it decreases exponentially, whereas in QV it approaches a finite constant (see Fig. $1)$.

(iii) At intermediate frequencies, the phase shift between $\widetilde{V}_{\mathrm{xc}}^{\mathrm{ALDA}}$ and $V_{\mathrm{xc}}^{M}$ varies between $\pi$ and $\pi / 2$, where $\pi / 2$ indicates a purely dissipative potential (see below).

(iv) The average strength of the forces associated with

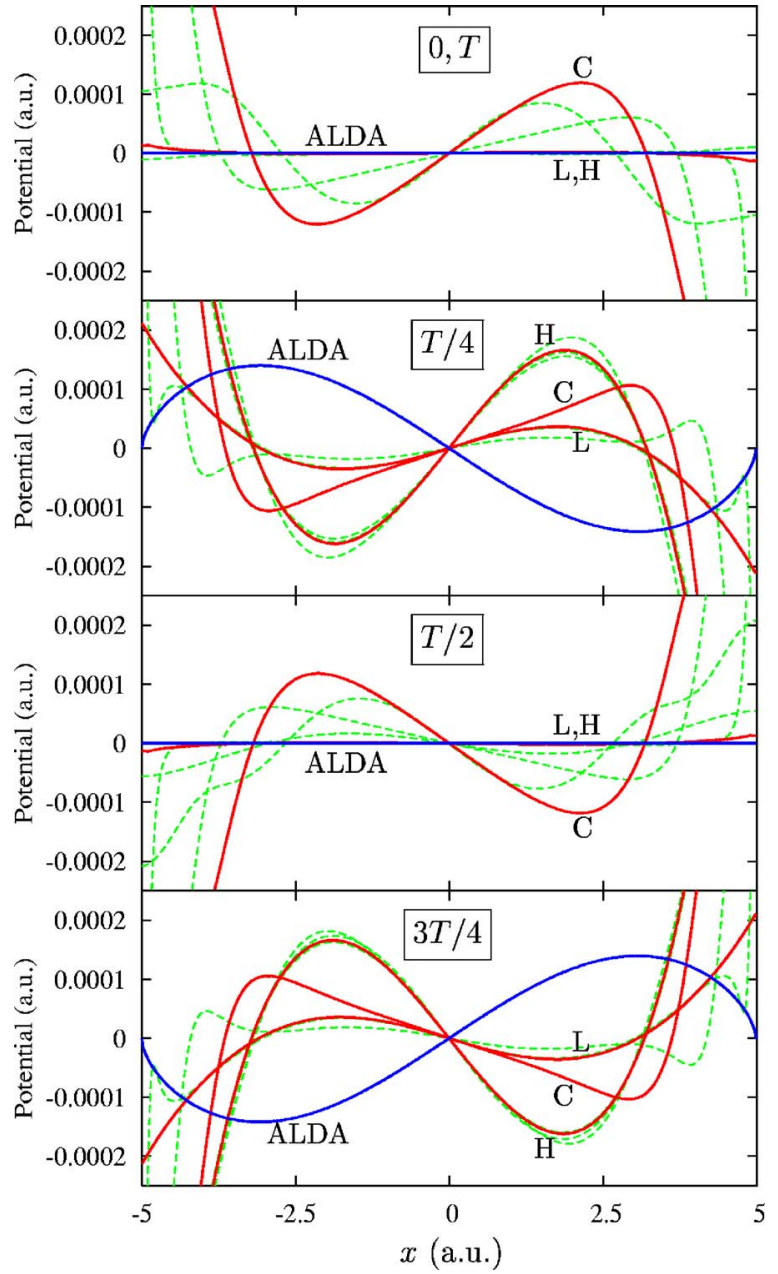

FIG. 5. (Color online) The same as Fig. 4, but $V_{\mathrm{xc}}^{M}$ calculated in QV parametrization. The crossover frequency is $\omega_{C}=2.15 \bar{\omega}_{p}$.

$V_{\mathrm{xc}}^{M}$ grows with frequency, and becomes comparable to the ALDA fluctuating forces in the high-frequency limit (notice that $\widetilde{V}_{\mathrm{xc}}^{\mathrm{ALDA}}$ is scaled by 0.1 in Figs. 4 and 5). This clearly shows that nonadiabatic effects can become non-negligible in practice. We will say more about this below when we discuss the nonlinear regime.

\section{Power and dissipation}

For a more quantitative analysis, it is useful to consider the power associated with the charge-density oscillations of the two types of modes. We define the power in the usual way as xc force density times velocity:

$$
\mathcal{P}(t)=\int d x v(x, t) n(x, t) \frac{\partial}{\partial x} V_{\mathrm{xc}}^{M}(x, t) .
$$

This expression can also be derived microscopically as the rate of change of the Kohn-Sham energy. ${ }^{28}$ Figure 8 shows $\mathcal{P}(t)$, scaled by $\omega A^{2}$, during one cycle of the sloshing and breathing modes (calculated using the GK parametrization). In the low-frequency limit, where the currents are vanishing, the power tends to zero, but as the frequency increases, a periodic input/output of power is observed to take place dur- 


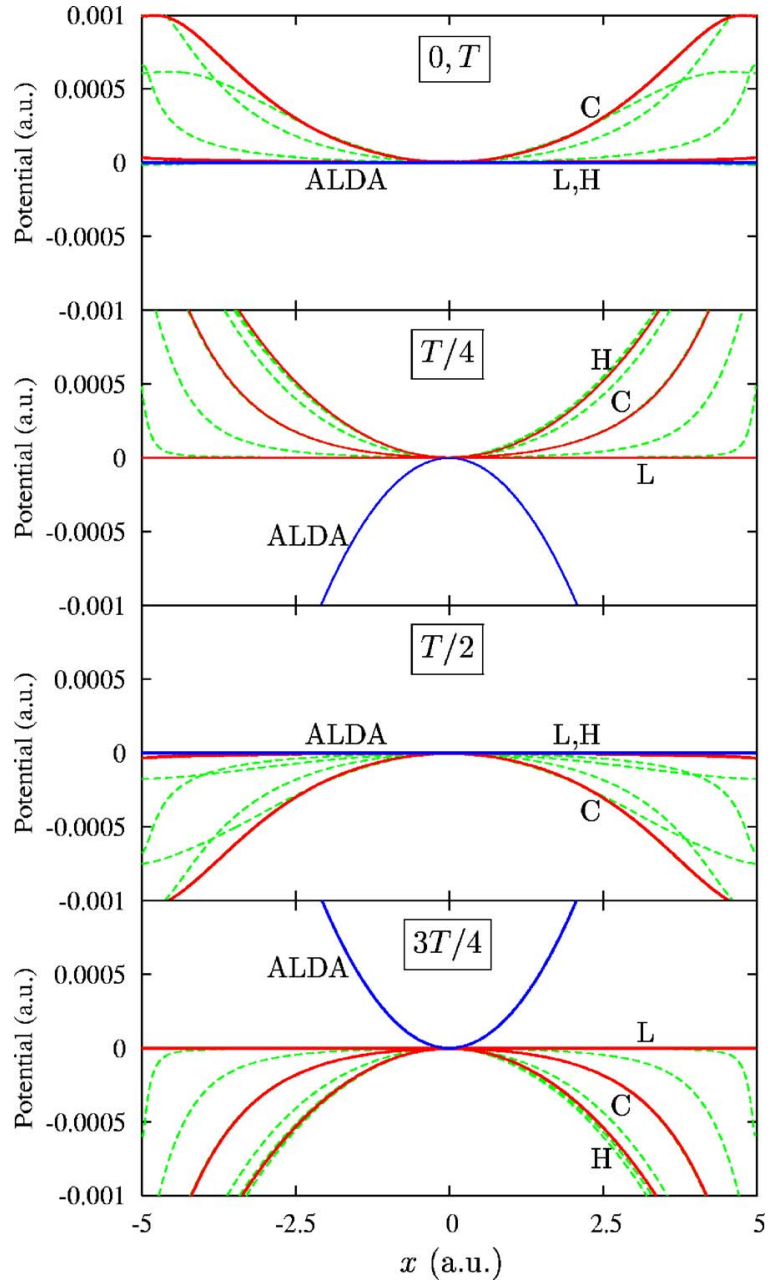

FIG. 6. (Color online) The same as Fig. 4, but for the breathing mode (Fig. 3). $\widetilde{V}_{\mathrm{xc}}^{\mathrm{ALDA}}$ is not scaled. The crossover frequency is $\omega_{C}=2.22 \bar{\omega}_{p}$.

ing a cycle. It can be clearly seen that, on average, $\mathcal{P}$ is more negative than positive for intermediate frequencies, which indicates net power dissipation. In the high-frequency, elastic limit, $\mathcal{P}(t)$ has sizable amplitudes, but averages to zero during a cycle.

These findings are summarized in Figs. 9 and 10. We plot $-\overline{\mathcal{P}}$, the absolute value of the time average of $\mathcal{P}(t)$, scaled by $\omega A^{2}$, which represents the net power absorption per cycle, for the sloshing and the breathing modes. Notice that $-\overline{\mathcal{P}}$ in all cases has a pronounced enhancement for frequencies of the order of $\bar{\omega}_{p}$. We define the crossover frequencies $\omega_{C}$ as those frequencies where the maxima of power absorption occur.

While the two modes behave qualitatively very similarly as far as their power absorption is concerned, one observes in Figs. 8-10 that the absorbed power of the breathing mode is about an order of magnitude higher than for the sloshing mode, for the same value of the amplitude, $A=0.005$. This hardly comes as a surprise: our sloshing mode can be viewed as a cousin of Kohn's mode, ${ }^{35,36}$ and the Harmonic Potential Theorem $^{29}$ tells us that charge-density oscillations in parabolic quantum wells are undamped. The breathing mode, on

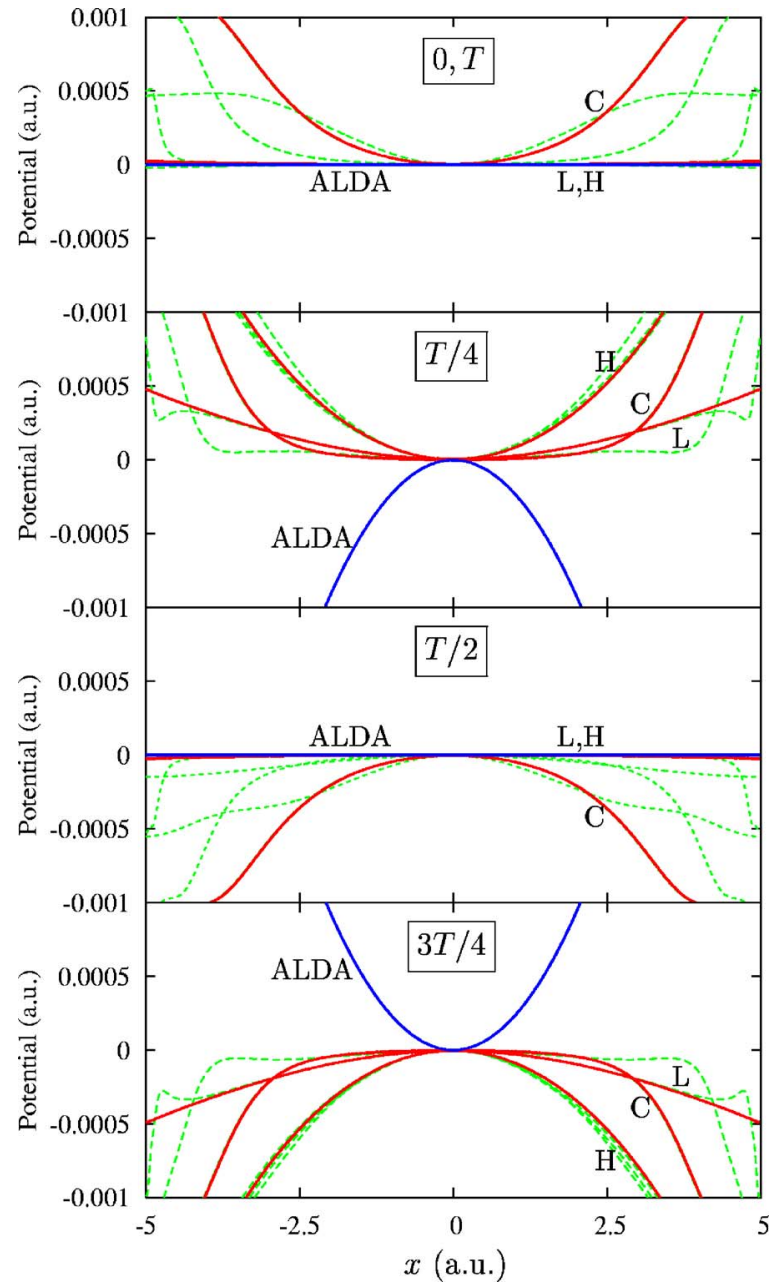

FIG. 7. (Color online) The same as Fig. 6, but $V_{\mathrm{xc}}^{M}$ calculated in QV parametrization. The crossover frequency is $\omega_{C}=2.65 \bar{\omega}_{p}$.

the other hand, bears no resemblance at all to Kohn's mode. The sloshing mode has a smooth hydrodynamic flow with relatively little internal compression, except at the turning points. By contrast, the defining feature of the breathing mode is the periodic compression and rarefaction of its density profile, i.e., a very "unhydrodynamic" behavior.

It turns out that there is a direct relation between the average power absorption and the imaginary part of the xc kernel, $\Im f_{\mathrm{xc}}^{L}(\omega)$. This is clear from Eq. (19), which expresses the memory kernel $Y\left(n, t-t^{\prime}\right)$ via the Fourier transform of $\Im f_{\mathrm{xc}}^{L}(\omega) / \omega$. The full lines in Figs. 9 and 10 plot $-\gamma \mathfrak{I} f_{\mathrm{xc}}^{L}(\omega)$, where $\gamma$ is a constant scaling factor. For a best fit, $\gamma_{\mathrm{sl}}$ $=0.00525$ and $r_{s}^{\mathrm{sl}}=1.41$ for the sloshing mode, and $\gamma_{\mathrm{br}}$ $=0.071$ and $r_{s}^{\mathrm{br}}=1.18$ for the breathing mode (the same for GK and QV). Notice that the equilibrium density $n_{0}(x)$ has a value of $r_{s}=1.06$ in the center. It thus emerges that the dissipation is dominated by different regions of the density distribution for the two modes: around $x= \pm 0.27 \mathrm{~L}$ for the sloshing mode, i.e., halfway between the center and the walls, and $x= \pm 0.18 L$ for the breathing mode, i.e., much closer to the center.

The phase lag of the modes as a function of frequency was already discussed in the context of Figs. 4-7. However, 


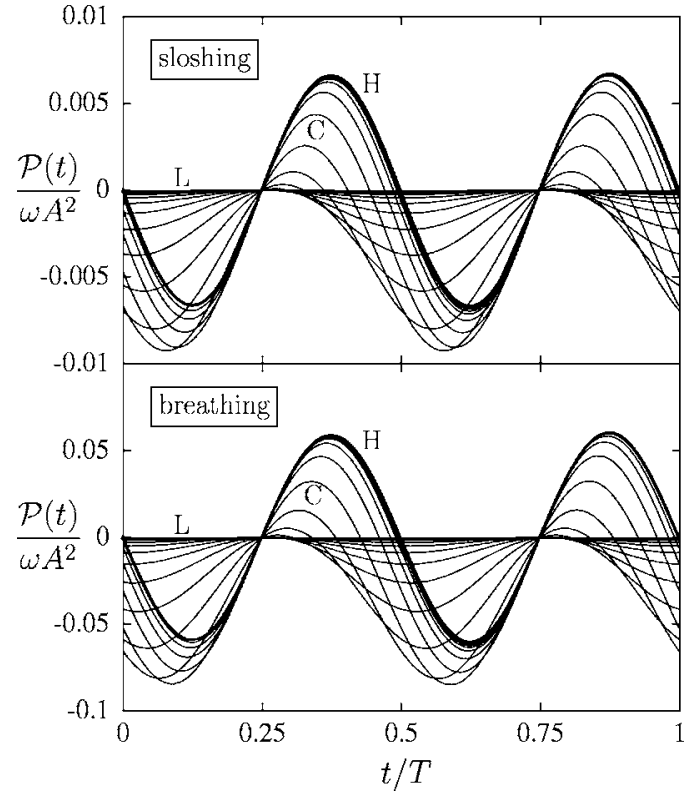

FIG. 8. Power $\mathcal{P}(t)$ [Eq. (58)] calculated using GK, associated with the sloshing and the breathing mode, during one cycle of the charge-density oscillation $(T=2 \pi / \omega)$, for different frequencies. $L$, $C, H$ indicate the low-frequency (purely dissipative in GK), crossover, and high-frequency regime (purely elastic).

in Figs. 9 and 10 one can see the difference between GK and QV most dramatically. We consider the phase lag between the instantaneous power absorption, $\mathcal{P}(t)$, and the ALDA potential fluctuations (which are in phase with the charge-

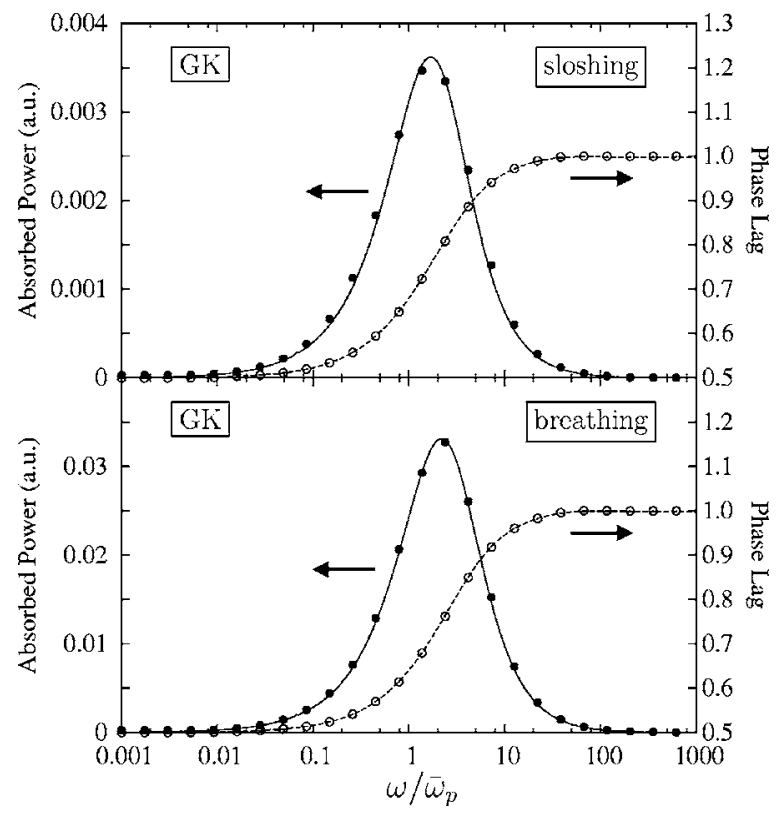

FIG. 9. Full symbols: net rate of power absorption, $-\overline{\mathcal{P}} / \omega A^{2}$, during one cycle of the sloshing and breathing modes, versus frequency (in GK). Full line: $-\gamma \Im f_{\mathrm{xc}}^{L}(\omega)$, for $r_{s}=1.41$ (sloshing) and 1.18 (breathing). Open symbols: Phase lag (in units of $\pi$ ) between $\mathcal{P}(t)$ and ALDA. The crossover frequency is defined by a maximum in power absorption and a phase lag $3 \pi / 4$. The dashed lines are a guide to the eye.

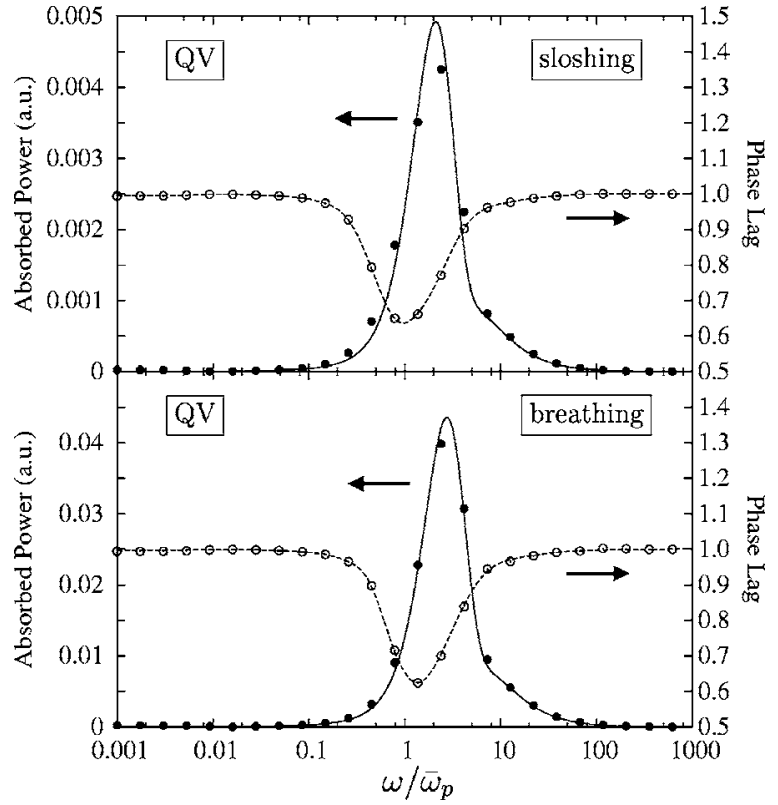

FIG. 10. The same as Fig. 9, but in QV parametrization. Both the low- and high-frequency limits are purely elastic.

density oscillations). Figure 9 shows that in GK, one has a transition from a purely dissipative behavior in the lowfrequency limit, with phase difference $\pi / 2$, via a crossover region of mixed dissipative/elastic behavior, to the highfrequency, purely elastic regime with phase lag $\pi$. The maximal power absorption occurs for a phase lag of $3 \pi / 4$. On the other hand, in the QV parametrization the low-frequency regime is also purely elastic, i.e., both the low- and highfrequency limits have phase lag $\pi$, and dissipative contributions come in only at intermediate frequencies. The resulting power loss as a function of frequency is thus a bit higher and more narrowly peaked about $\omega_{C}$ in QV than in GK.

As mentioned earlier, the qualitative differences between GK and QV have their origin in the different long-time behavior of the memory kernels; see Fig. 1 and the discussion in Sec. II A 2. The overall result is that there is a broad range of frequencies, between about 0.1 to 10 times the characteristic average plasma frequency $\bar{\omega}_{p}$, where the system exhibits a mixed elastic/dissipative behavior, which can lead to substantial dissipation.

\section{B. Nonlinear regime}

\section{C-vs L-TDDFT in the high-frequency limit}

In the following, we will extend our numerical studies of $\mathrm{C}$ - and L-TDDFT into the nonlinear regime. We begin by directing our attention to the high-frequency, purely elastic region, since our approximate version of L-TDDFT becomes exact in that limit and can thus be used as a benchmark to assess the accuracy of nonlinear C-TDDFT.

Figure 11 shows the scaled power $\mathcal{P}(t) / \omega A^{2}$ [Eq. (58)] for $\omega=10^{4} \widetilde{\omega}_{p}$ during one cycle of the sloshing and breathing modes, comparing small amplitudes $(A=0.005)$ and large amplitudes $(A=0.5$ and 0.75$)$. In the small-amplitude regime, $\mathcal{P}(t) / \omega A^{2}$ has a sinusoidal shape for both modes, indepen- 


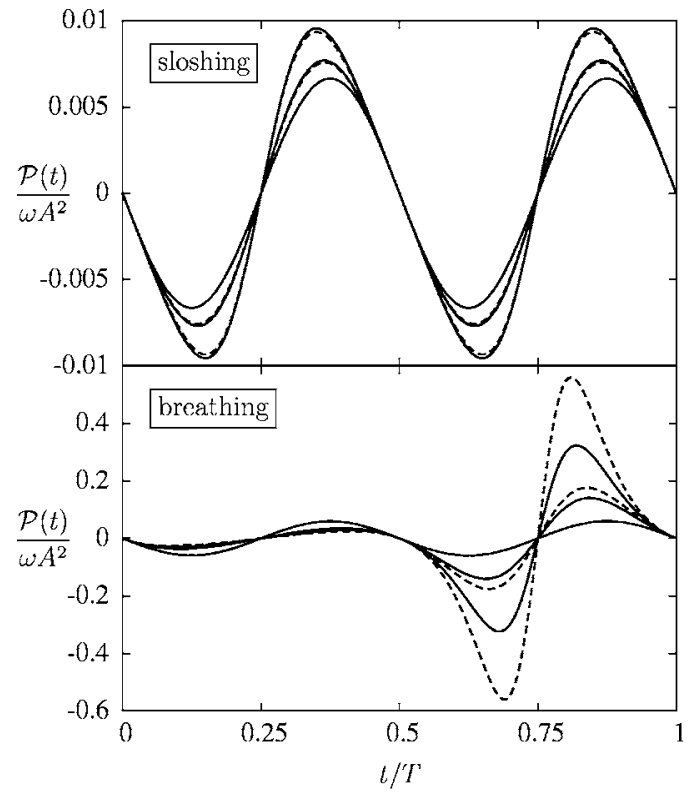

FIG. 11. Power $\mathcal{P}(t)$ [Eq. (58)] calculated using GK, over one cycle of the sloshing and the breathing modes, for $\omega=10^{4} \widetilde{\omega}_{p}$. Full and dashed lines: C- and L-TDDFT for amplitudes $A=0.005,0.5$, and 0.75. At $A=0.005, \mathrm{C}$ - and L-TDDFT are identical (see Fig. 8).

dent of the amplitude $A$ of the oscillations (see also Fig. 8), but for large amplitudes, nonlinear deviations occur. In the sloshing mode, $\mathcal{P}(t)$ tends to a more sawtoothlike shape. In the breathing mode, $\mathcal{P}(t)$ becomes suppressed when the charge density spreads out in the first half of the cycle, and dramatically enhanced when the density gets squeezed in the second half of the cycle. As we will see later, these strong deformations give rise to sizable nonadiabatic effects.

For small amplitude, $A=0.005, \mathrm{C}$ - and L-TDDFT are identical, but for larger amplitudes, $A=0.5$ and 0.75 , some differences develop. However, the magnitude of these deviations strongly depends on the type of mode. For the sloshing mode, we find that C-TDDFT remains quite close to L-TDDFT, even for large-amplitude oscillations, whereas in the breathing mode, C-TDDFT deviates from L-TDDFT by about a factor of 2 for large values of $A$.

A more quantitative measure of the deviation of C-TDDFT from the exact high-frequency L-TDDFT result is shown in Fig. 12. We plot the difference in percent of the time average of the absolute value of the scaled power, $\left(t_{2}\right.$ $\left.-t_{2}\right)^{-1} \int_{t_{1}}^{t_{2}} d t|\mathcal{P}(t)| / \omega A^{2}$, over one cycle for the sloshing mode, and over the first and second half-cycle for the breathing mode, as a function of the amplitude $A$. As expected, the difference is seen to be increasing as the amplitude grows, but the two modes exhibit a very different behavior.

Due to its close kinship to Kohn's mode, it comes as no surprise that the deviation is much smaller for the sloshing mode, namely at most around $2 \%$, even for very large amplitudes. The C-TDDFT error of the breathing mode is larger by at least an order of magnitude. However, the overall error of C-TDDFT in the high-frequency limit compared to L-TDDFT remains surprisingly small, as long as the amplitude is not too large: For $A=0.2$, we get a deviation of about $0.2 \%$ for the sloshing mode, and about $5 \%$ for the breathing

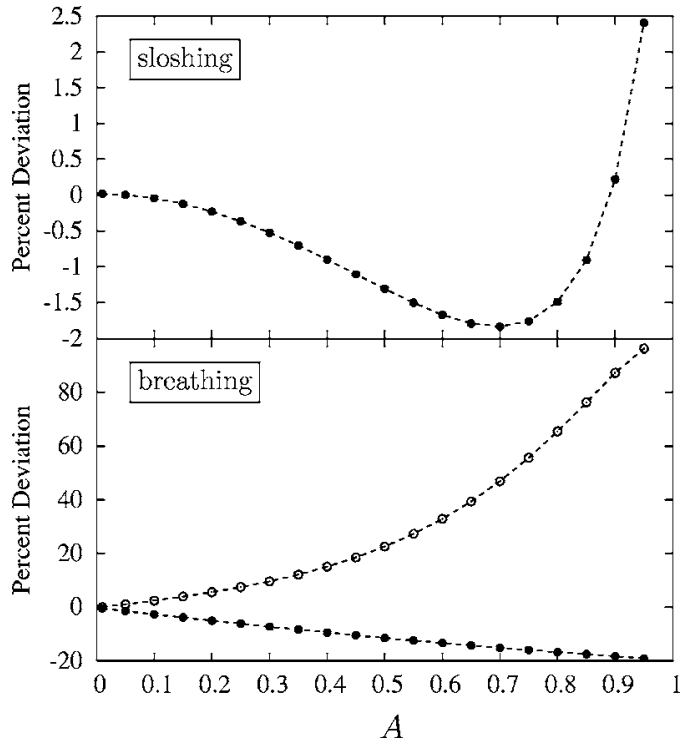

FIG. 12. Percent deviation of C-TDDFT from the (exact) L-TDDFT result for the time-averaged absolute value of the scaled power, $\left(t_{2}-t_{2}\right)^{-1} \int_{t_{1}}^{t_{2}} d t|\mathcal{P}(t)| / \omega A^{2}$. Top panel: sloshing mode, averaged over one cycle. Bottom panel: breathing mode, averaged over the first (full symbols) and the second half-cycle (open symbols).

mode. For the largest amplitude considered $(A=0.9)$, we obtain a deviation of $2.5 \%$ for the sloshing mode, and $20 \%$ and $100 \%$ for first and second half-cycles of the breathing mode. In the latter case, the deformations are so strong as to invalidate the basic assumptions used to derive the simple form of nonlinear C-TDDFT.

\section{Nonadiabatic corrections to the ALDA}

The preceding high-frequency analysis shows that the C-TDDFT nonadiabatic xc potentials remain close (to within a few percent) to the exact L-TDDFT results, except for modes with extremely strong deformations such as sloshing modes with $A \gtrsim 0.9$ or breathing modes with $A \gtrsim 0.2$. As long as the deformations remain within these approximate limits, it is reasonable to expect C-TDDFT to be accurate for finite frequencies as well.

To get an impression of the magnitude of the nonadiabatic corrections to the ALDA, we plot in Figs. 13 and 14 the full adiabatic and nonadiabatic xc potentials, $V_{\mathrm{xc}}^{\mathrm{ALDA}}(x, t)$ and $V_{\mathrm{xc}}^{\mathrm{ALDA}}(x, t)+V_{\mathrm{xc}}^{M}(x, t)$, where $V_{\mathrm{xc}}^{M}$ is calculated within $\mathrm{C}$-TDDFT using the QV parametrization. Figure 13 shows results for a large-amplitude sloshing mode with $A=0.75$, and Fig. 14 for a breathing mode with $A=0.5$. The figures show four snapshots taken during one cycle of the mode, similar to what was presented in Figs. 4-7, except that now we are plotting the total potential. The ALDA xc potential is of course independent of the mode frequency, and we compare it with the ALDA $+M$ potential at three different frequencies: $\omega=0.1 \widetilde{\omega}_{p}$ (low-frequency regime), $\omega=\widetilde{\omega}_{p}$ (around crossover), and $\omega=10 \widetilde{\omega}_{p}$ (approaching the high-frequency regime).

The results in Figs. 13 confirm again that the nonadiabatic effects for the sloshing mode are relatively modest, even for 


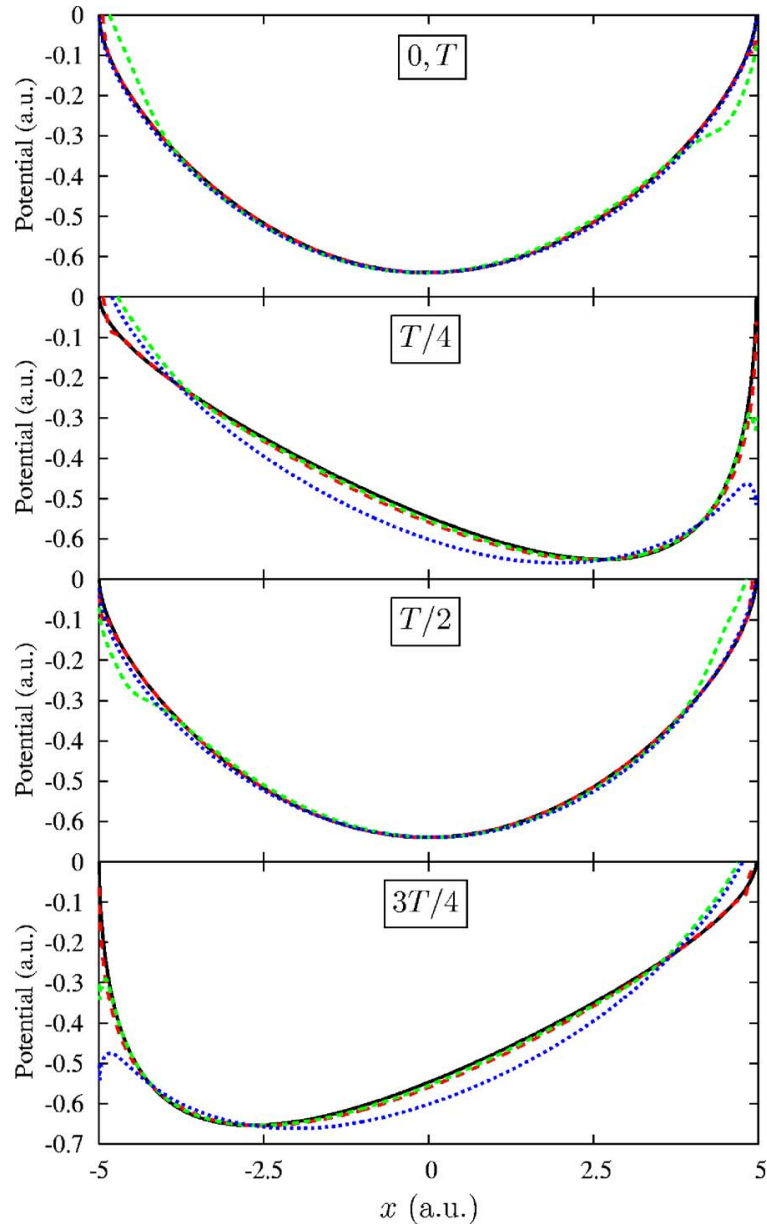

FIG. 13. (Color online) Snapshots of the adiabatic and nonadiabatic xc potentials during one cycle of the sloshing mode, with amplitude $A=0.75$. Black solid line: $V_{\mathrm{xc}}^{\mathrm{ALDA}}$. Red (medium gray) long-dashed, green (light gray) medium-dashed, and blue (dark gray) dotted lines: $V_{\mathrm{xc}}^{\mathrm{ALDA}}+V_{\mathrm{xc}}^{M}$ for frequencies $\omega=0.1 \widetilde{\omega}_{p}, \omega=\widetilde{\omega}_{p}$, and $\omega=10 \widetilde{\omega}_{p}$.

large-amplitude deformations. The post-ALDA corrections become more important for the high-frequency oscillations $\left(\omega=10 \widetilde{\omega}_{p}\right)$, where one finds deviations from ALDA of the order of $10 \%$ at the turning points of the oscillation at $T / 4$ and $3 T / 4$. For the lower-frequency modes, the nonadiabatic corrections to the ALDA stay mostly within about $1 \%$.

On the other hand, the breathing mode exhibits much more dramatic nonadiabatic effects; see Fig. 14. Again, we find that the post-ALDA corrections are moderate for the lower frequencies considered. However, for the highfrequency case $\left(\omega=10 \widetilde{\omega}_{p}\right)$, we find that at the instances of maximum deformation (T/4 and 3T/4) the memory effects cause a correction to the ALDA of up to a factor of 2 , which is indeed substantial.

The impact of the high-frequency post-ALDA corrections is similar for both modes: they tend to oppose the deformation of the ALDA potential at the instances of maximum deformation of the density distribution. For the breathing mode, this means that $V_{\mathrm{xc}}$ becomes less broad at $T / 4$ and less deep at $3 T / 4$, and for the sloshing mode that the potential minimum lies closer to the center, and is less sharp. In a

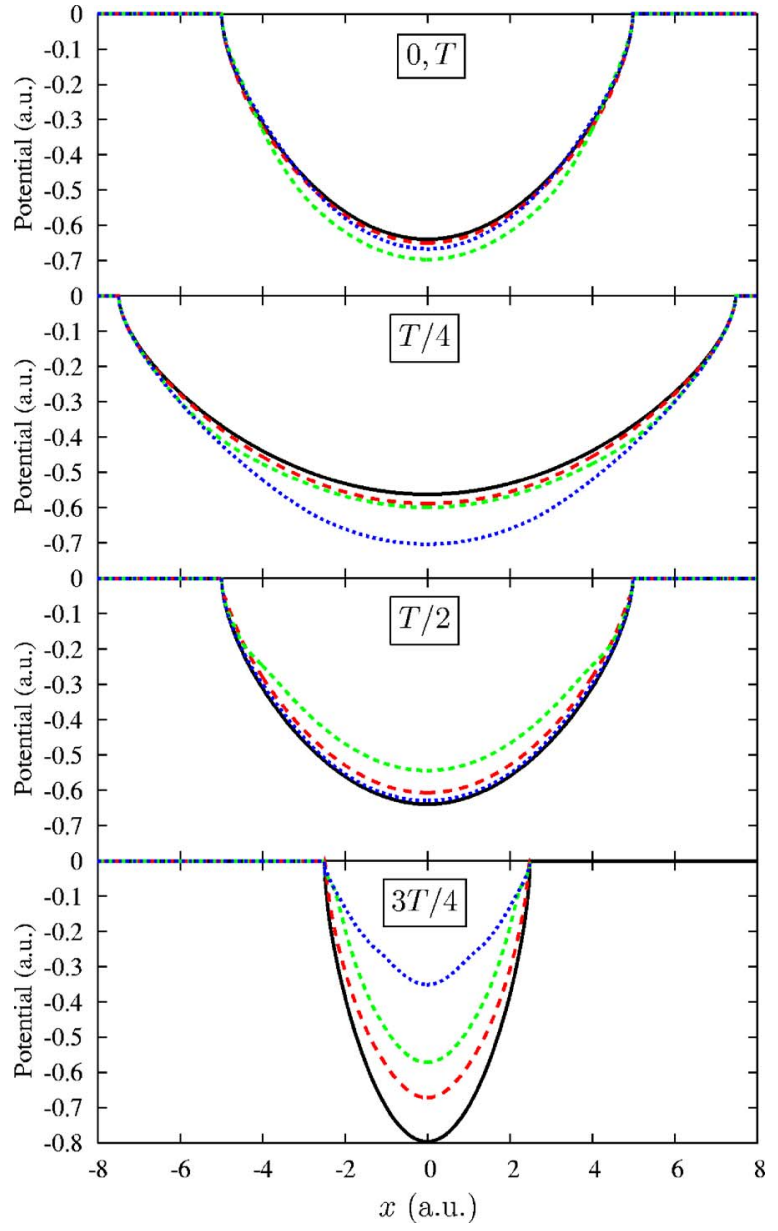

FIG. 14. (Color online) The same as Fig. 13, but for the breathing mode with amplitude $A=0.5$.

more realistic calculation, where the density, instead of being a given function, follows from solving a TDKS calculation with a time-dependent driving potential, this would imply that the elasticity of the electron liquid tends to counteract deformations of the density, making the system more rigid and somewhat harder to deform.

Lastly, to illustrate a case of extreme nonadiabaticity let us consider the high-frequency limit of strongly nonlinear dynamics. Figure 15 shows a comparison of the ALDA and L-TDDFT xc potentials for both modes with amplitude $A$ $=0.75$, for the same snapshots taken during one cycle, as previously in Figs. 13 and 14. For the instants $0, T / 2$, and $T$, ALDA and L-TDDFT coincide, but for $T / 4$ and 3T/4, large deviations occur. The most dramatic nonadiabatic effect is observed for the breathing mode at $3 T / 4$, where the elastic contribution of L-TDDFT is so large that the resulting xc potential is of the same magnitude as the ALDA, but with opposite sign. This clearly shows that in situations in which the electron density is rapidly and strongly deformed the ALDA becomes drastically wrong, with nonadiabatic corrections of the same order of magnitude as the potential itself.

\section{CONCLUSION}

In this paper, we have carried out a detailed comparative study of two nonadiabatic local approximations for the $\mathrm{xc}$ 

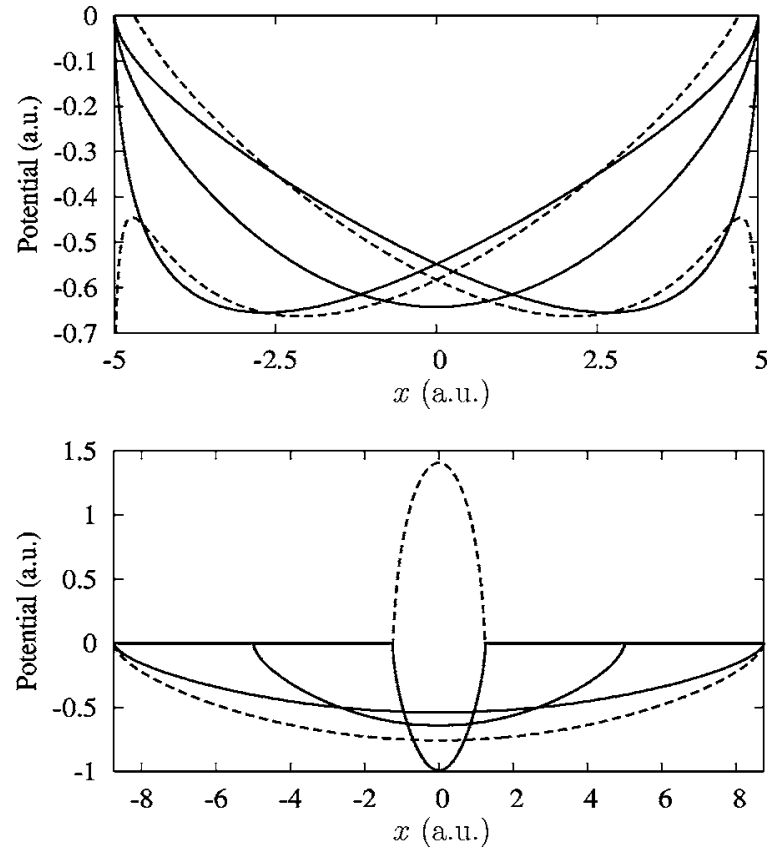

FIG. 15. Comparison of ALDA (full lines) and L-TDDFT (dashed lines) xc potentials for the sloshing mode (top panel) and the breathing mode (bottom panel), both with amplitude $A=0.75$. The same snapshots during one cycle are shown as in Figs. 13 and 14.

potential in TDDFT, using quasi-one-dimensional model systems (whose electronic density is uniform in two spatial directions, and nonuniform in the third). The goal was to compare the $\mathrm{xc}$ potentials resulting from $\mathrm{C}$-TDDFT and L-TDDFT when a given time-dependent density is used as input, and to determine the magnitude of the resulting nonadiabatic effects when compared to the ALDA. These comparisons were carried out for two types of collective modes: a sloshing and a breathing mode, for a wide dynamical range of amplitudes and frequencies.

In our discussion of nonadiabatic xc effects, we were paying particular attention to dynamical regimes where the two theories, C-TDDFT and elastic L-TDDFT, are known to be exact: C-TDDFT in the linear regime of small-amplitude oscillations, regardless of the frequency, and elastic L-TDDFT in the high-frequency limit, for oscillations of an arbitrary amplitude. This helps to shed light on the so far open question concerning the validity of C-TDDFT in the nonlinear regime: it turns out that, at least in the high-frequency limit, C-TDDFT is surprisingly accurate. Our results show that for moderate deformations (up to $20 \%$ of the initial density distribution) errors in the xc potential remain within a few percent. This gives us good reason to believe that this nonlinear formalism should work well for finite frequencies, too (as long as the deformations are not too large).

Let us now address the fundamental question of the meaning and the importance of "nonadiabatic effects" in TDDFT, based on the insights resulting from our study. In analyzing these effects, the language of hydrodynamics, as it is used in C- and L-TDDFT, proves very useful. In general, nonadiabaticity manifests itself through elastic and dissipative components of the electron dynamics, which can be distinguished by their characteristic phase lag with respect to the ALDA. Both elastic and dissipative effects enter the theory via the dependence of xc potential on the deformation tensor. Since the deformation is defined relative to the initial state, this dependence reflects how much of its history the system carries in its memory. A local-in-time dependence on the deformation, which occurs in the high-frequency regime, corresponds to an extremely pronounced memory and a purely elastic xc potential. The dissipative contribution formally appears in the form of a time nonlocality in the dependence of the xc potential on deformations of the electron fluid. In general, this time-nonlocality tends to shorten the characteristic memory time, and, as a result, it somewhat diminishes the elastic contribution. In addition, it brings about fundamentally new effects, such as relaxation and the corresponding power absorption. Which of the two contributions (elastic or dissipative) is dominant, or whether both play an equally important role, depends on the dynamical regime in which the system under study is evolving. The results from our simple model system lead us to the following conclusions.

Linear regime. For small-amplitude oscillations, dissipation is the most important and dramatic consequence of nonadiabaticity, which leads to qualitative corrections to the adiabatic dynamics. There is a dynamical range that we call the "crossover" regime, in which the power absorption due to $\mathrm{xc}$ retardation effects is maximal. This crossover regime occurs for frequencies that are comparable to an average plasmon frequency for the system. On the other hand, for very low or for very high frequencies, dissipation vanishes. Elastic effects in the linear regime appear to be less important, in a sense that they do not qualitatively change an overall behavior of the xc potential. However, the quantitative effect of elastic corrections to the linearized ALDA can be significant, especially in the high-frequency regime. In particular, for our breathing mode the post-ALDA nonadiabatic corrections are in general of the same order of magnitude as the dynamic part of the ALDA potential itself.

Nonlinear regime. Here, elastic effects become more important, especially at high frequencies, which can lead to substantial, and in certain regimes absolutely dominant contributions to the time-dependent xc potential. As we discussed for the sloshing and breathing modes, the elasticity of the electron liquid tends to oppose attempts to subject the electron system to strong and rapid deformations. This general tendency also naturally explains why C-TDDFT has been successful for molecular polarizabilities, which are greatly overestimated in ALDA. ${ }^{22}$ Dynamic polarization of the system corresponds to a redistribution of the charge density, i.e., to the deformation of an electron subsystem, which causes a counteracting xc force. This force is an intrinsically nonadiabatic effect that is completely missing in ALDA.

Our results once again illustrate the special role of Kohn's mode in TDDFT. If the electron dynamics sufficiently resembles Kohn's mode, as is the case for the sloshing mode considered here, nonadiabatic effects are generally small. On the other hand, for electron dynamics involving high degrees of compression, such as our breathing mode, the nonadiabatic "corrections" can become several times larger than the ALDA potential itself, so that the ALDA completely breaks 
down, leading to a qualitatively wrong behavior. A striking illustration of such a situation was shown in Fig. 15.

Strictly speaking, most of the discussions in this paper, especially those related to dissipative effects, are relevant only for nonadiabaticity in extended systems, such as our model densities (which are confined along $x$, but extended along $y$ and $z$ ). The purely electronic dissipation in TDDFT then has a well-defined physical meaning, ${ }^{28}$ as discussed in the Introduction. The situation is less clear when one attempts to describe nonadiabatic effects in finite systems such as atoms and molecules. ${ }^{25}$ Here, the elasticity of the electron liquid leads to small shifts of excitation energies, but one also obtains finite linewidths due to dissipation, which is clearly an unwanted effect. The question of how nonadiabatic xc potentials for small systems should be constructed thus remains an open issue.

\section{ACKNOWLEDGMENTS}

C. A. U. acknowledges support from NSF Grant No. DMR-0553485 and from the Research Corporation. We thank Giovanni Vignale for fruitful discussions.
${ }^{1}$ E. Runge and E. K. U. Gross, Phys. Rev. Lett. 52, 997 (1984).

${ }^{2}$ E. K. U. Gross and W. Kohn, Adv. Quantum Chem. 21, 255 (1990)

${ }^{3}$ E. K. U. Gross, J. F. Dobson, and M. Petersilka, in Density Functional Theory II, Vol. 181 of Topics in Current Chemistry (Springer-Verlag, Berlin, 1996), p. 81.

${ }^{4}$ R. van Leeuwen, Int. J. Mod. Phys. B 15, 1969 (2001).

${ }^{5}$ N. T. Maitra, K. Burke, and C. Woodward, Phys. Rev. Lett. 89, 023002 (2002).

${ }^{6}$ E. K. U. Gross and W. Kohn, Phys. Rev. Lett. 55, 2850 (1985); 57, 923(E) (1986).

${ }^{7}$ M. E. Casida, in Recent Advances in Density Functional Methods, edited by D. P. Chong (World Scientific, Singapore, 1995), p. 155.

${ }^{8}$ F. Furche and R. Ahlrichs, J. Chem. Phys. 117, 7433 (2002).

${ }^{9}$ G. Giuliani and G. Vignale, Quantum Theory of the Electron Liquid (Cambridge University Press, Cambridge, 2005).

${ }^{10}$ N. T. Maitra, F. Zhang, R. J. Cave, and K. Burke, J. Chem. Phys. 120, 5932 (2004).

${ }^{11}$ N. T. Maitra, J. Chem. Phys. 122, 234104 (2005).

${ }^{12}$ G. Vignale and W. Kohn, Phys. Rev. Lett. 77, 2037 (1996).

${ }^{13}$ G. Vignale, C. A. Ullrich, and S. Conti, Phys. Rev. Lett. 79, 4878 (1997).

${ }^{14}$ C. A. Ullrich and G. Vignale, Phys. Rev. B 65, 245102 (2002); 70, 239903(E) (2004).

${ }^{15}$ J. F. Dobson, M. J. Bünner, and E. K. U. Gross, Phys. Rev. Lett. 79, 1905 (1997).

${ }^{16}$ I. V. Tokatly and O. Pankratov, Phys. Rev. B 67, 201103(R) (2003).

${ }^{17}$ I. V. Tokatly, Phys. Rev. B 71, 165104 (2005).

${ }^{18}$ I. V. Tokatly, Phys. Rev. B 71, 165105 (2005).

${ }^{19}$ Y. Kurzweil and R. Baer, J. Chem. Phys. 121, 8731 (2004).

${ }^{20}$ C. A. Ullrich and G. Vignale, Phys. Rev. B 58, 15756 (1998).

${ }^{21}$ C. A. Ullrich and G. Vignale, Phys. Rev. Lett. 87, 037402 (2001).

${ }^{22}$ M. van Faassen, P. L. de Boeij, R. van Leeuwen, J. A. Berger, and J. G. Snijders, Phys. Rev. Lett. 88, 186401 (2002); J. Chem. Phys. 118, 1044 (2003).

${ }^{23}$ M. van Faassen and P. L. de Boeij, J. Chem. Phys. 120, 8353
(2004).

${ }^{24}$ J. A. Berger, P. L. de Boeij, and R. van Leeuwen, Phys. Rev. B 71, 155104 (2005).

${ }^{25}$ C. A. Ullrich and K. Burke, J. Chem. Phys. 121, 28 (2004).

${ }^{26}$ H. O. Wijewardane and C. A. Ullrich, Appl. Phys. Lett. 84, 3984 (2004).

${ }^{27}$ H. O. Wijewardane and C. A. Ullrich, Phys. Rev. Lett. 95, 086401 (2005).

${ }^{28}$ R. D’Agosta and G. Vignale, Phys. Rev. Lett. 96, 016405 (2006).

${ }^{29}$ J. F. Dobson, Phys. Rev. Lett. 73, 2244 (1994).

${ }^{30}$ R. Nifosi, S. Conti, and M. P. Tosi, Phys. Rev. B 58, 12758 (1998).

${ }^{31}$ Z. Qian and G. Vignale, Phys. Rev. B 65, 235121 (2002).

${ }^{32}$ In the most general formulation of L-TDDFT (Ref. 18), the exact xc stress tensor appears as a functional of the Cauchy's deformation tensor $\bar{g}_{i j}$, and a skew-symmetric vorticity tensor $F_{i j}$. However, in the lowest order of the gradient expansion the dependence on $F_{i j}$ disappears (for details see Ref. 18), so that $P_{\mathrm{xc}, i j}$ becomes a local functional of only one tensor variable, $\bar{g}_{i j}$.

${ }^{33}$ The sign difference reflects an ambiguity in the definition of the stress tensor in the continuum mechanics literature. The standard formulation of C-TDDFT (Ref. 13) follows the sign convention used in the classical elasticity theory, where the divergence of the stress tensor $\sigma_{i j}$ defines a force exerted on a small volume element by surroundings parts of the body. In contrast, L-TDDFT in its original formulation (Refs. 17 and 18) accepts another convention that is more common in fluid mechanics and in microscopic theory. Here the sign of the stress tensor $P_{i j}$ coincides with the sign of momentum flow tensor. With this definition the trace of $P_{i j}$ is equal to the local pressure, while its divergence gives the force exerted by an infinitesimal volume element on a surrounding fluid.

${ }^{34}$ S. Conti and G. Vignale, Phys. Rev. B 60, 7966 (1999).

${ }^{35}$ W. Kohn, Phys. Rev. 123, 1242 (1961).

${ }^{36}$ L. Brey, N. F. Johnson, and B. I. Halperin, Phys. Rev. B 40, 10647 (1989); L. Brey, J. Dempsey, N. F. Johnson, and B. I. Halperin, ibid. 42, 1240 (1990); S. K. Yip, ibid. 43, 1707 (1991). 